\title{
Analysis of the transverse heat transfer coefficients in a dual channel ITER-type cable-in-conduit conductor
}

L. Bottura, P. Bruzzone, C. Marinucci,

B. Stepanov 



\title{
Analysis of the transverse heat transfer coefficients in a dual channel ITER-type cable-in-conduit conductor
}

\author{
Luca Bottura $^{\mathrm{a}}$, Pierluigi Bruzzone ${ }^{\mathrm{b}}$, Claudio Marinucci ${ }^{\mathrm{b}}$, Boris Stepanov ${ }^{\mathrm{b}}$ \\ ${ }^{a}$ CERN, AT-MAS, 1211 Geneva 23, Switzerland \\ b EPFL, CRPP, 5232 Villigen PSI, Switzerland
}

CRPP/SC/CM/2006/01

20th September 2006

\begin{abstract}
This report describes a new method to determine the equivalent heat transfer coefficients in CICC's with parallel cooling channels, i.e. the radial heat transfer coefficient between helium flow in the cable bundle and in the central spiral, and the azimuthal heat transfer coefficient between subcables in the bundle. The method is based on the Fourier analysis of the steady state temperature traces during a heat step experiment after calibration of the thermometers. The equations for the average temperature distributions in the cable are solved analytically and the values of the equivalent transverse heat transfer coefficients are obtained as the best fit of the experimental temperature distributions. We show the results of the method by application to a short length sample experiment in the SULTAN test facility using an ITER-type CICC. The special instrumentation includes thermometers to measure the temperature in the center of the conductor and at 6 locations equally spaced in angle around the periphery of the conductor jacket, at 3 cross sections along the sample length. Heathers of different geometry allows generating a variety of heat slugs.
\end{abstract}




\section{Contents}

1 Introduction 3

2 Experimental set-up $\quad 5$

2.1 Conductor and sample layout $\ldots \ldots \ldots \ldots \ldots \ldots$

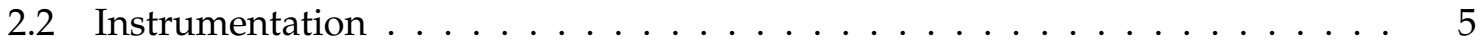

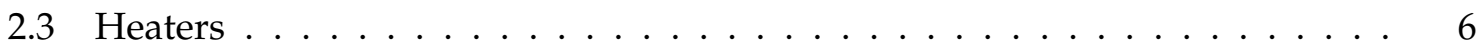

3 Experiments and calibration $\quad 11$

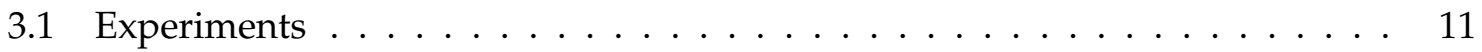

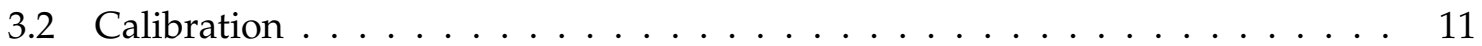

4 Measurement analysis $\quad 20$

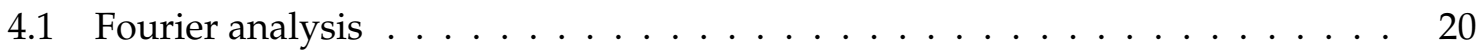

4.2 Axial temperature distribution $\ldots \ldots \ldots \ldots \ldots \ldots$

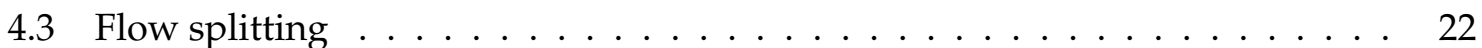

5 Discussion 33

5.1 Azimuthal heat transfer coefficient . . . . . . . . . . . . . . . . 33

5.2 Radial heat transfer coefficient . . . . . . . . . . . . . . . . . . 33

5.3 Sensitivity analysis $\ldots \ldots \ldots \ldots \ldots \ldots \ldots \ldots$

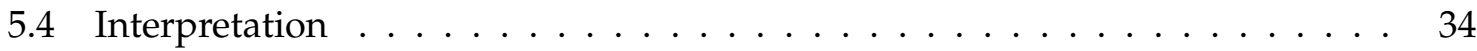

6 Conclusions $\quad 42$

$\begin{array}{ll}\text { References } & 43\end{array}$

Appendix A. Analytical solution $\ldots \ldots \ldots \ldots \ldots$

Appendix B. Fourier analysis . . . . . . . . . . . . . . . . . . 56

Appendix C. Porous media analogy _ . . . . . . . . . . . . . . . 57

List of Symbols . . . . . . . . . . . . . . . . . . . . . . . 63

List of Tables and List of Figures $\ldots \ldots \ldots$. . . . . . . . . . . . . 65 


\section{Introduction}

A superconducting sample, referred to as the "Low Cost Joint" (LCJ), was developed at CRPP with the specific aim to investigate the thermal hydraulic properties of its conductor $[1,2]$. The LCJ conductor is an ITER ${ }^{1}$-type dual channel cable-in-conduit conductor (CICC). In this particular type of conductor the coolant flows in parallel in the central channel (subscript $\mathrm{H}$ for hole) and in the annular bundle region (subscript B) (Fig. 1 and 2). The LCJ sample was tested in the CRPP SULTAN Facility in 2005. The main goal was to assess the transverse heat transfer coefficients, namely the azimuthal heat transfer coefficient between subcables in the bundle $\left(\mathrm{h}_{B B}\right)$ and the radial heat transfer coefficient between bundle and central channel $\left(\mathrm{h}_{B H}\right)$.

We describe here the special instrumentation of the LCJ sample [3] that has been used to derive $\mathrm{h}_{B B}$ and $\mathrm{h}_{B H}$ using a different approach than the one used to analyze another dual channel CICC also tested in SULTAN (ITER PFIS sample) [4]. The results of this analysis provide reliable scaling indications and typical values of the transverse heat transfer coefficients in spite of the presence of heater and thermometer in the LCJ central channel as described below.

Experimental set-up, experiments, calibration, measurement analysis, results, discussion, and conclusions are described in the next Sections [5]. Details of the theory, i.e. analytical solution, the Fourier analysis and the porous media analogy, are given in Appendix A, B and C, respectively [6]. Lists of Symbols, Tables and Figures are shown at the end.

\footnotetext{
${ }^{1}$ International Thermonuclear Experimental Reactor.
} 


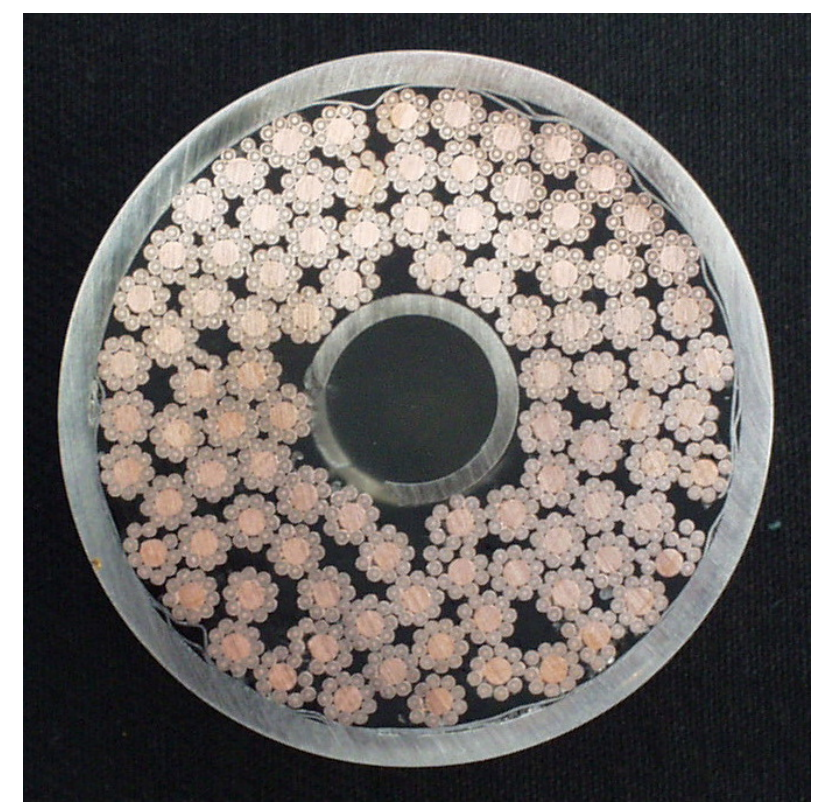

Figure 1: Cross section of the Low Cost Joint conductor.

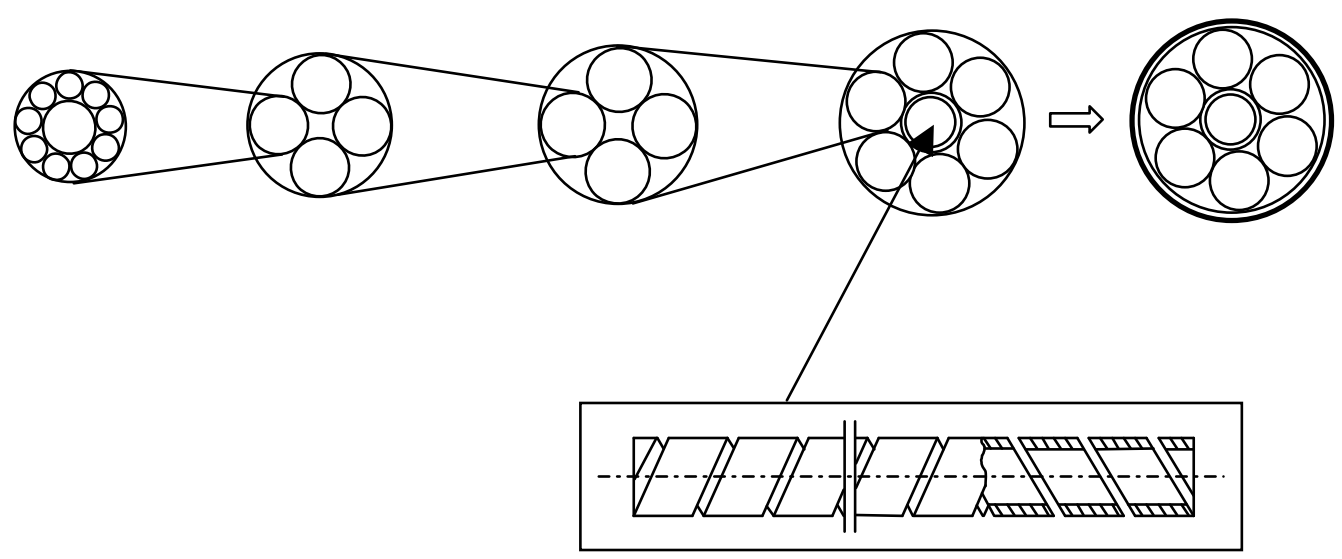

Figure 2: Schematic build up of the Low Cost Joint conductor, with details of the central spiral. 


\section{Experimental set-up}

\subsection{Conductor and sample layout}

The LCJ cable-in-conduit conductor was manufactured in the Russian Federation ${ }^{2}$. It includes $864 \mathrm{NbTi}$ strands of $0.73 \mathrm{~mm}$ diameter. The cabling pattern is $(1+9) \times 4 \times 4 \times 6$ and the twist pitch of the last stage (right helix) is $\mathrm{L}_{\text {last }}=350 \mathrm{~mm}$. The diameter of the segregated copper core is $1.45 \mathrm{~mm}$. The outer diameters of conductor and cable space are $39.50 \mathrm{~mm}$ and $35.95 \mathrm{~mm}$, respectively. The central channel is a spiral whose inner and outer diameter are $9.40 \mathrm{~mm}$ and $11.40 \mathrm{~mm}$, respectively. The $2 \mathrm{~mm}$ thick jacket is made of $316 \mathrm{LN}$ stainless steel. After compaction, the void fraction in the bundle $\left(v f_{B}\right)$ is $38.31 \%$. The conductor has outer cable wraps (stainless steel, $0.1 \mathrm{~mm}$ thick) and no subcable wraps. Geometric parameters of the LCJ conductor and other data derived for this analysis are listed in Table 1.

The LCJ sample consists of two conductor "legs" of $\approx 3.5 \mathrm{~m}$ in vertical orientation. Electrical and hydraulic connections of the two conductors is provided by the hairpin joint at the bottom of the sample. The overlap joint is included on the left leg. The direction of the helium flow is from bottom to top (Fig. 3).

\subsection{Instrumentation}

Only the right leg of the sample has the special instrumentation for thermal hydraulic (TH) measurements [3]. This consists of thermometers distributed on three sensor rings (R1, R2 and R3) at three different locations along the sample axis, i.e. $X 1=1350 \mathrm{~mm}, X 2=1850 \mathrm{~mm}$, and $X 3=2330 \mathrm{~mm}$, where $X$ is the coordinate from the sample inlet. Each location includes a total of 7 temperature sensors (Fig. 3):

- Six Cernox sensors are evenly spaced along the circumference of the conductor jacket. The notation for the respective temperature is $T_{i j}$, where $i$ is the index for the 3 rings and $j$ the index for the 6 sensors. Each sensor monitors the temperature of one subcable along its twist pitch. For example, the sensor $T_{11}$ and $T_{21}$ are located at azimuth angles $\phi_{1}=0^{\circ}$ and $\phi_{2}=154^{\circ}$, where $\phi_{2}-\phi_{1}=360^{\circ} /\left((X 2-X 1) / L_{\text {last }}\right)$. The sensors are nested on a special supporting ring which is tightened to the jacket (Fig. 4). Thermal contact between sensor and jacket is obtained by Apiezon $\mathrm{N}$ grease.

- One Cernox sensor is located in the helium, inside the central channel $\left(T_{i 7}\right)$, at the same cross section as the three rings.

A mass flow sensor near the helium outlet and a thermometer at the helium inlet $\left(T_{0}\right)$ are also part the $\mathrm{TH}$ instrumentation.

\footnotetext{
${ }^{2}$ VNIINM-Moscow for the strands, VNIIKP-Podolosk for cabling and jacketing.
} 


\subsection{Heaters}

Heat deposition into the conductor is provided by 5 resistive heaters of different size, located at different positions along the sample length (Fig. 3), which allow generating a variety of heat slugs.

- Heaters in helium. This group includes the main helium heater H0 located upstream and far away from the sample inlet, and the heater $\mathrm{H} 7$, which is inserted in the central channel $(X=1280 \mathrm{~mm})$ (Fig. 6).

- Heaters wrapped on the full conductor jacket circumference, providing uniform heat deposition in the azimuthal direction. This group includes the annular heaters H3 $(X=520 \mathrm{~mm})$ and $\mathrm{H} 9(X=1280 \mathrm{~mm})$.

- Heater distributed only on a limited part of the conductor jacket providing localized heat deposition, i.e. $\mathrm{H} 8$ at $\mathrm{X}=1210 \mathrm{~mm}$. 
Table 1: Geometric parameters used for the thermal hydraulic analysis of the LCJ sample. The data in the first part of the Table are from the specifications ([1], [2]), those in the second part are deduced.

\begin{tabular}{lccc}
\hline Description & Symbol & Value & Unit \\
\hline Conductor outer diameter & $d_{c o n}$ & 39.50 & $\mathrm{~mm}$ \\
Cable space diameter & $d_{c s}$ & 35.95 & $\mathrm{~mm}$ \\
Strand diameter & $d_{s t}$ & 0.73 & $\mathrm{~mm}$ \\
Number of strands & $n_{s t}$ & 864 & - \\
Cabling parameter & $\cos \theta$ & 0.96 & - \\
Spiral inner diameter & $d i n_{s p}$ & 9.40 & $\mathrm{~mm}$ \\
Spiral outer diameter & $d_{\text {out }}$ & 11.40 & $\mathrm{~mm}$ \\
Spiral perforation & $p_{\text {spiral }}$ & 25 & $\%$ \\
Wrap thickness & $t_{\text {ow }}$ & 0.10 & $\mathrm{~mm}$ \\
\hline Total helium in bundle & $A_{B t o t}$ & 349.7665 & $\mathrm{~mm}^{2}$ \\
Helium in central channel (hole) & $A_{H}$ & 69.3978 & $\mathrm{~mm}^{2}$ \\
Hydraulic diameter of bundle & $D_{B}$ & 0.7494 & $\mathrm{~mm}$ \\
Hydraulic diameter of hole (1) & $D_{H}$ & 11.40 & $\mathrm{~mm}$ \\
Wetted perimeter bundle/bundle (2) & $p_{B B}$ & 12.2750 & $\mathrm{~mm}$ \\
Total wetted perimeter bundle/hole $(2)$ & $p_{B H t o t}$ & 35.8141 & $\mathrm{~mm}$ \\
Void fraction of bundle & $v f_{B}$ & 38.3104 & $\%$ \\
\hline
\end{tabular}

Notes:

(1) $D_{H}=$ dout $_{s p}$

(2) Calculated from geometric values: $p_{B B}=\left(d_{c s}-d o u t_{s p}\right) / 2$ and $p_{B H t o t}=\pi d o u t_{s p}$ 




Figure 3: Schematic experimental set-up of the LCJ sample in SULTAN. Helium enters the sample from the bottom, is heated up by 5 resistive heaters ( $\mathrm{H} 0, \mathrm{H} 3, \mathrm{H} 7, \mathrm{H} 8, \mathrm{H} 9)$ and the warm front propagates along the sample length towards the outlet at the top. Only the right leg is used for the thermal hydraulic measurements. The 21 temperature sensors $\left(T_{11}-\right.$ $\left.T_{17} \ldots T_{31}-T_{37}\right)$ at three rings along the sample (R1, R2 and R3) are shown. The area near the heaters H7, H8 and $\mathrm{H} 9$ is enlarged for clarity. The distance between the heater $\mathrm{H} 0$ and the sample inlet is not to scale. The mass flow rate sensor near the sample outlet is not shown. The thermometer $T_{28}$, clamped on sample (not at R2) and used to monitor the cool down, is not shown. 


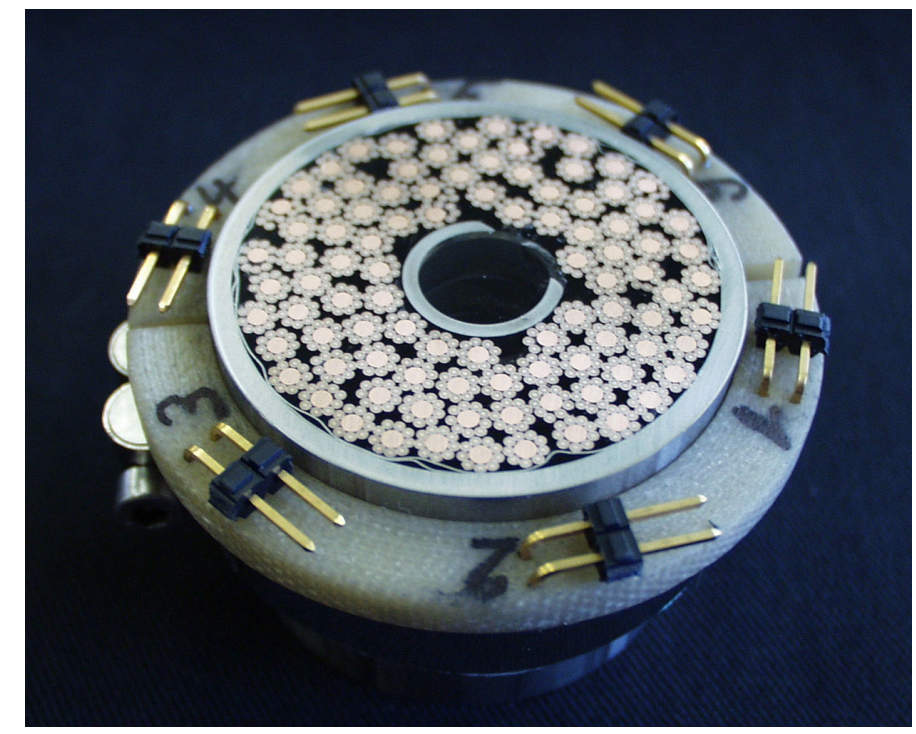

Figure 4: Sensor ring applied on the LCJ conductor.

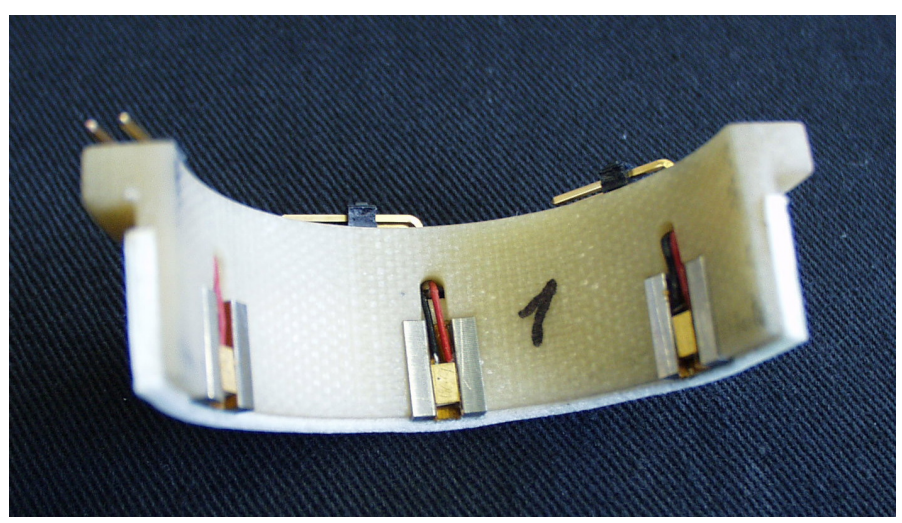

Figure 5: Details of one half of the sensor ring with 3 Cernox sensors. 


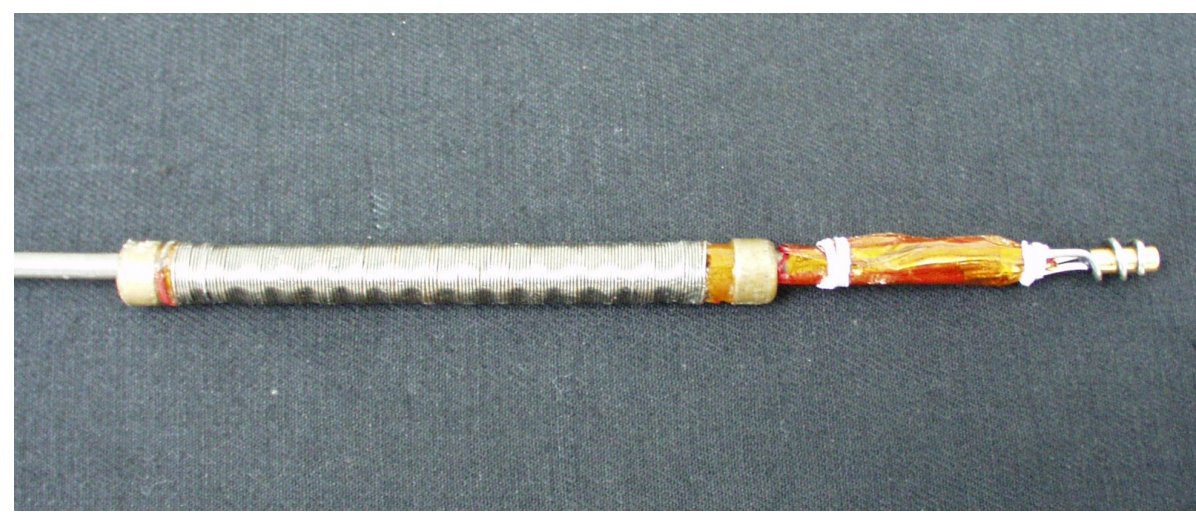

Figure 6: Helium heater H7 (left) and thermometer $T_{17}$ (right) which are included in the central spiral. 


\section{Experiments and calibration}

\subsection{Experiments}

All thermal hydraulic measurements are performed at zero SULTAN background magnetic field $\left(B_{S U L T A N}\right)$ and zero current in the sample $\left(I_{L C J}\right)$. The helium initial conditions at the sample inlet are: $4.5 \mathrm{~K}$ and $1.0 \mathrm{MPa}$. The total helium mass flow rate $(\dot{m})$ is adjusted in the range between $2 \mathrm{~g} / \mathrm{s}$ and $8 \mathrm{~g} / \mathrm{s}$ at the sample outlet on the SULTAN facility side. All runs are listed in Table 2. Each TH measurement includes two heating procedures performed in sequence:

- After stable thermal hydraulic conditions are reached, the selected heater is switched on for a first time step until the helium outlet temperature has reached steady state condition at $t=t_{\text {end }}$. This analysis deals only with the first heating, i.e. steady state temperature evolution.

- After the heater is switched off and the temperatures have reached the new steady state conditions, the same heater is fired a second time for a duration of $3 \mathrm{~s}$. This procedure is then repeated two more times for 10s and 30s. The second heating procedure, i.e. transient temperature evolution, is not part of this analysis.

The results of a typical TH measurement (run LCJH280907, heater $\mathrm{H} 8, \dot{m}=8 g / s$ ), including steady state and transient evolutions, are shown as an example in Fig. 7; details of the first heating step deposition are shown in Fig. 8. In this run, as well as in all other runs, it can be observed that all sensors on the jacket give signals which are characterized by offsets of different size. This is due to inadequate thermalisation of the sensor leads because of the severe space restrictions.

\subsection{Calibration}

Special runs were used to calibrate the thermometers on the jacket, i.e. to offset the effect of poor thermalisation of the sensor leads. They were performed at the end of each of the three days of TH measurements, i.e. 27, 28 and 29 September 2005 (Table 2). The operating conditions are: $B_{S U L T A N}=0, I_{L C J}=0, \dot{m}=4 \mathrm{~g} / \mathrm{s}$ (the mass flow rate is irrelevant for the calibration of the thermometers). After steady state conditions are reached, the helium heater $\mathrm{HO}$ is fired in cascade five times, with steps of approximately the same duration. The raw measurements of a typical calibration run (LCJH280911) are shown in Fig. 9.

The heater H0 heats up the helium upstream of the inlet and therefore provides by definition uniform temperature everywhere along the sample, i.e. $T_{i 7}=T_{i j}$ for all $i$ and $j$. The helium temperatures $T_{17}, T_{27}$ and $T_{37}$ can be used as reliable reference to rescale all other signals using a linear fit. The relationship between raw temperature measurements $\left(T_{\text {ijraw }}\right)$, the reference temperature in the central channel $\left(T_{i 7}\right)$, and the corresponding calibrated tem- 
peratures $\left(T_{i j c a l}\right)$ is:

$$
T_{i 7}=T_{i j c a l}=p_{1}+p_{2} T_{i j r a w}
$$

where the coefficients $p_{1}$ and $p_{2}$ were obtained by least square fitting. An example of this linear fit (run LCJH280911, ring R1) is shown in Fig. 10.

The raw temperatures of all $\mathrm{TH}$ runs are calibrated with the above procedure, using the parameters $p 1$ and $p 2$, i.e one pair per day, ring and sensor. The error after re-scaling is < $50 \mathrm{mK}$, as shown in the example of the typical run LCJH280907 (Fig. 12). 
Table 2: Summary of thermal hydraulic runs (TH) and calibration runs of the LCJ sample. All runs are performed at $B_{S U L T A N}=0$ and $I_{L C J}=0$. The helium initial conditions at the sample inlet are: 4.5K and 1.0MPa. The runs LCJH270907, LCJH280911 LCJH290909 were used to calibrate all TH measurements of the same day. The results of heater $\mathrm{H} 3$ at $4 \mathrm{~g} / \mathrm{s}$ and $6 \mathrm{~g} / \mathrm{s}$ are not consistent with the other results of the measurement set and therefore have been rejected in this report.

\begin{tabular}{ccccc}
\hline Type & Heater & Run/Filename & $\dot{m}(\mathrm{~g} / \mathrm{s})$ & $\mathrm{t}_{\text {end }}(\mathrm{s})$ \\
\hline TH & H0 & LCJH270901 & 2 & 380.66 \\
& & LCJH270902 & 4 & 294.50 \\
& & LCJH270903 & 6 & 212.49 \\
& & LCJH270904 & 8 & 185.26 \\
\hline TH & H3 & LCJH280902 & 2 & 285.41 \\
& & LCJH270905 & 8 & 192.28 \\
\hline TH & H7 & LCJH280903 & 2 & 268.38 \\
& & LCJH280904 & 4 & 165.23 \\
& & LCJH280905 & 6 & 171.25 \\
TH & H8 & LCJH280906 & 8 & 127.18 \\
\hline & & LCJH280910 & 2 & 259.37 \\
& & LCJH280909 & 4 & 247.35 \\
TH & H9 & LCJH280907 & 8 & 185.26 \\
& & LCJH290901 & 2 & 153.22 \\
\hline & & LCJH290902 & 4 & 252.36 \\
& & LCJH290903 & 6 & 197.28 \\
& & LCJH290904 & 8 & 209.30 \\
\hline Calibration & H0 & LCJH270907 & 4 & - \\
& & LCJH280911 & 4 & - \\
& & LCJH290909 & 4 & - \\
\hline Note: First 4 digits in Run / Filename is date, e.g. 27=day, 09=month. \\
\hline
\end{tabular}



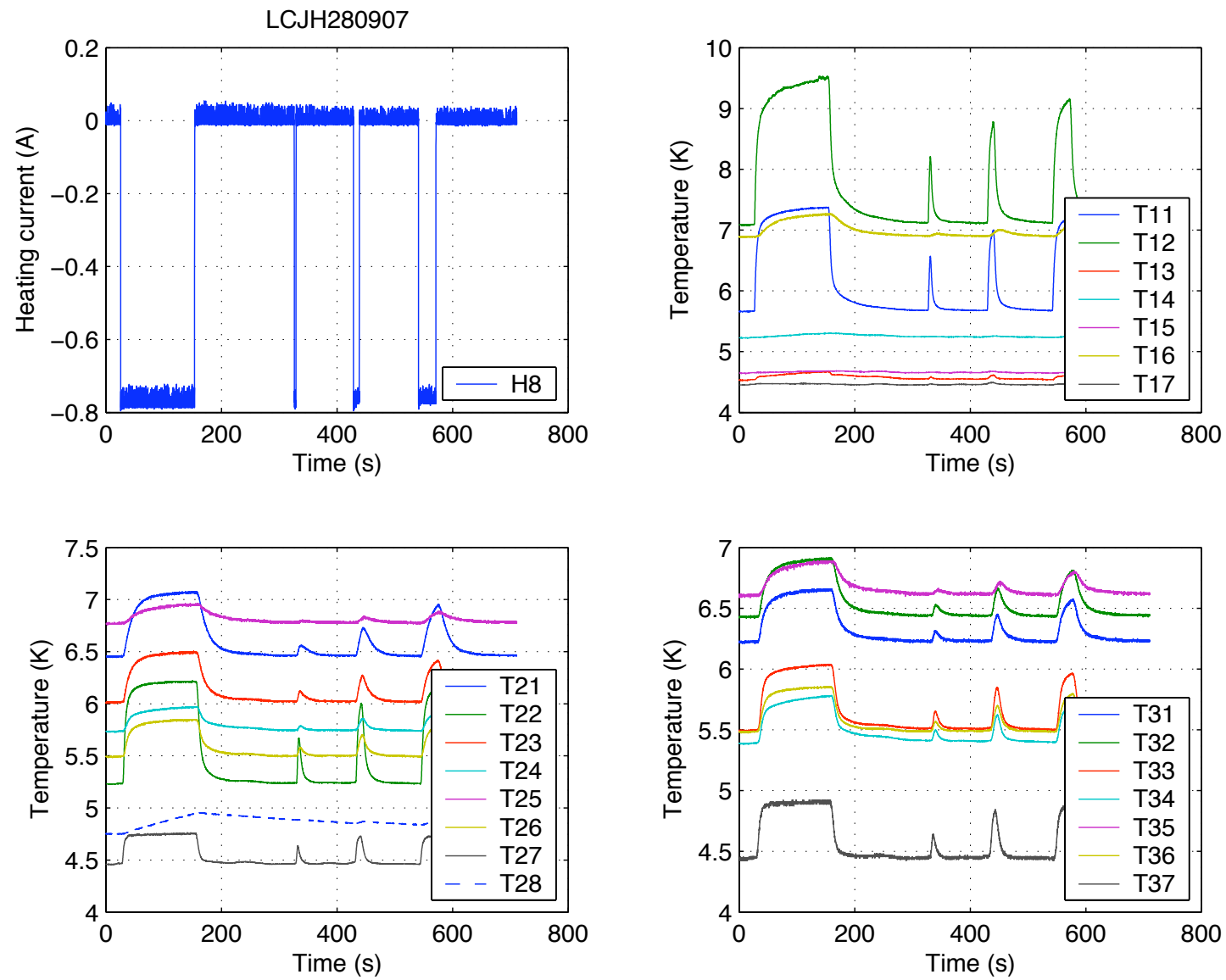

Figure 7: Example of a typical TH measurement before the temperature calibration, i.e. run LCJH280907, $\dot{m}=8 \mathrm{~g} / \mathrm{s}$, heater H8. Both the steady state (first step) and transient heating are shown. The time history of the heating current is shown in the upper-left plot. The time history of the raw temperatures in the rings R1, R2 and R3 are shown in the remaining three plots. The signal $T_{28}$ (lower left plot) is included for completeness but is not used in this analysis. 

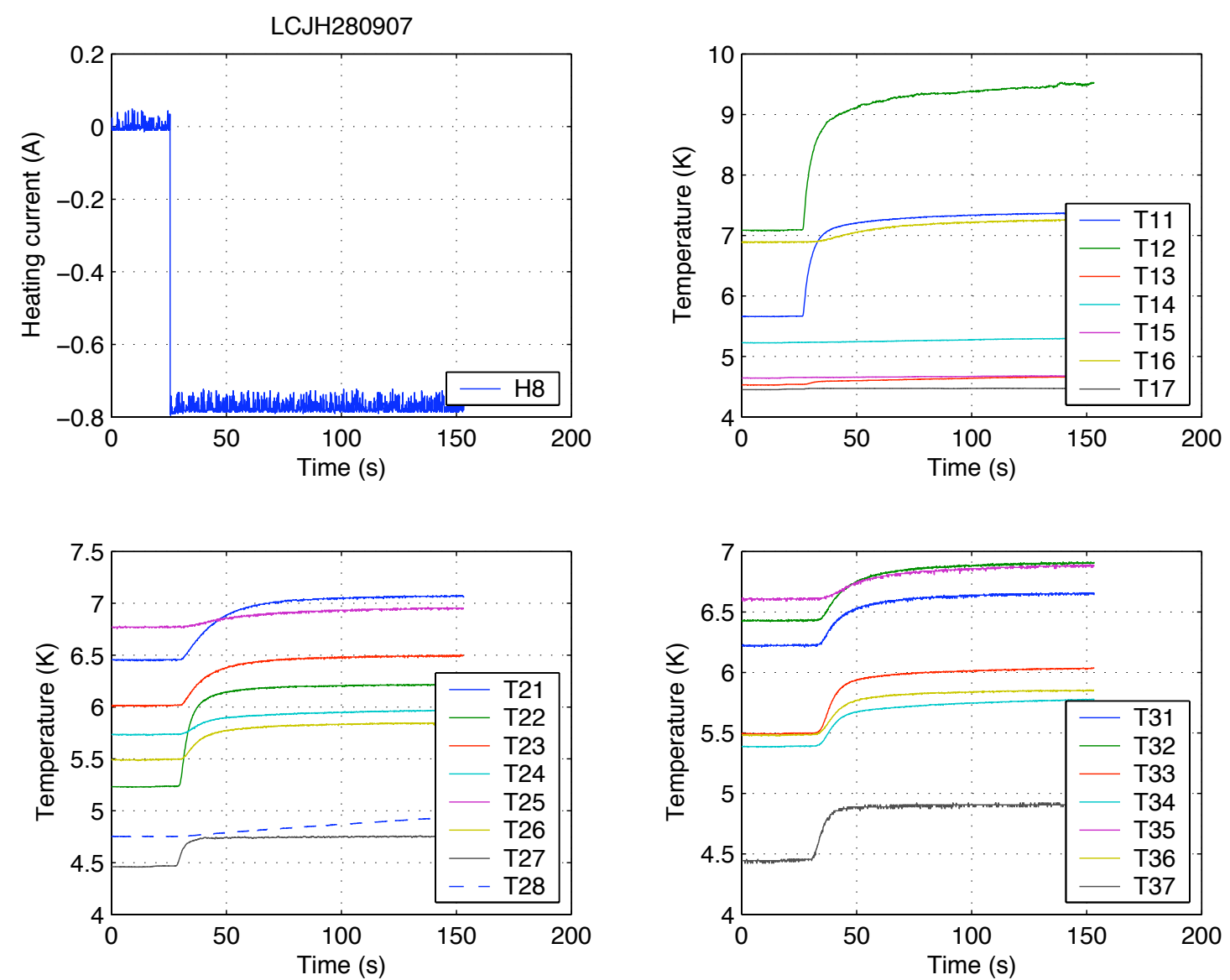

Figure 8: Example of a typical TH measurement before the temperature calibration, i.e. run $\mathrm{LCJH} 280907, \dot{m}=8 \mathrm{~g} / \mathrm{s}$, heater H8. Only the steady state (first step) heating is shown. The time history of the heating current is shown in the upper-left plot. The time history of the raw temperatures in the rings $\mathrm{R} 1, \mathrm{R} 2$ and $\mathrm{R} 3$ are shown in the remaining three plots. The signal $T_{28}$ (lower left plot) is included for completeness but is not used in this analysis. 

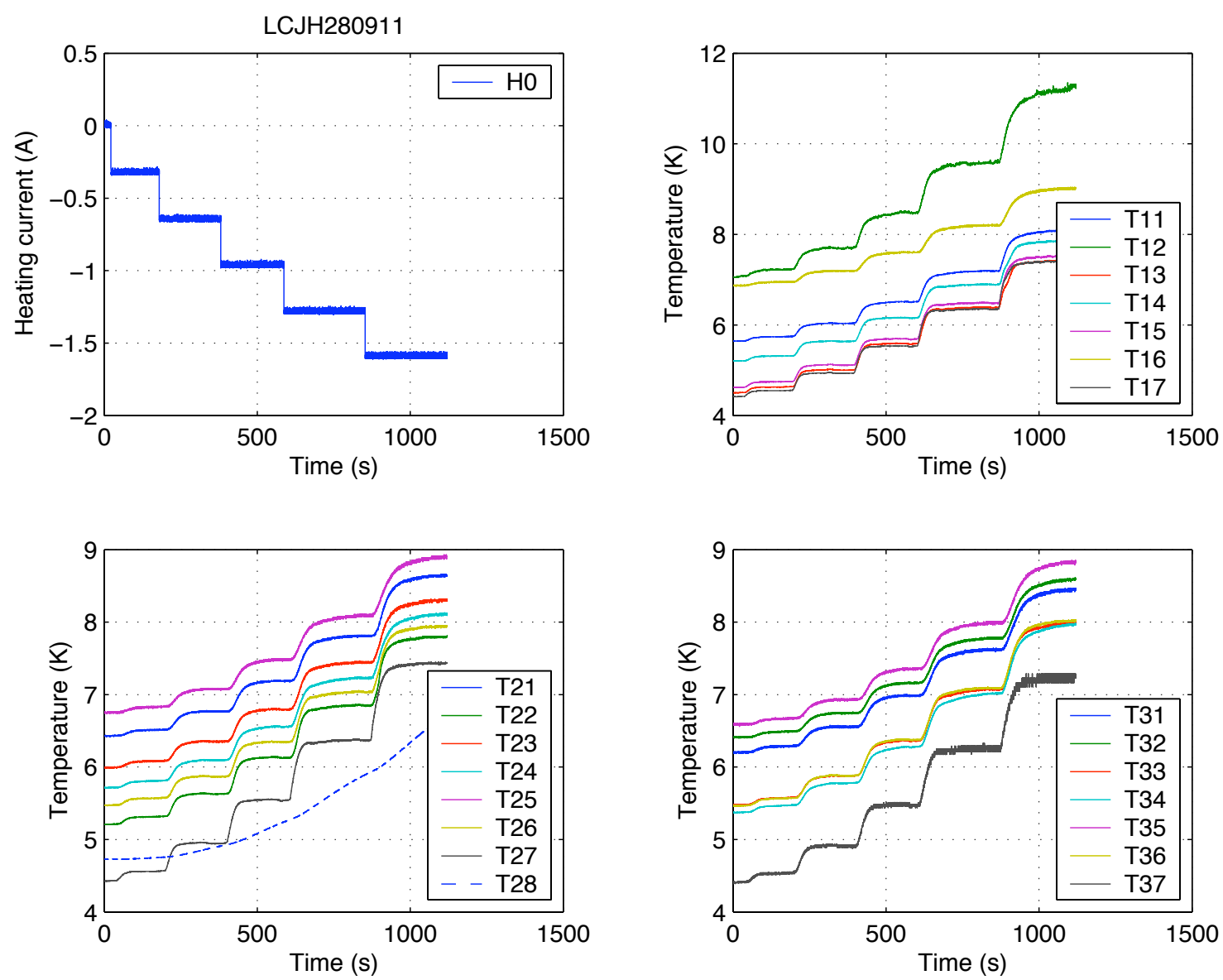

Figure 9: Example of a typical calibration run, i.e. LCJH280911, 28 September 2005. The time history of the heating current is shown in the upper-left plot. The time history of the raw temperatures at the rings R1, R2 and R3 are shown in the remaining three plots. The signal $T_{28}$ (lower left plot) is included for completeness but is not used in this analysis. 

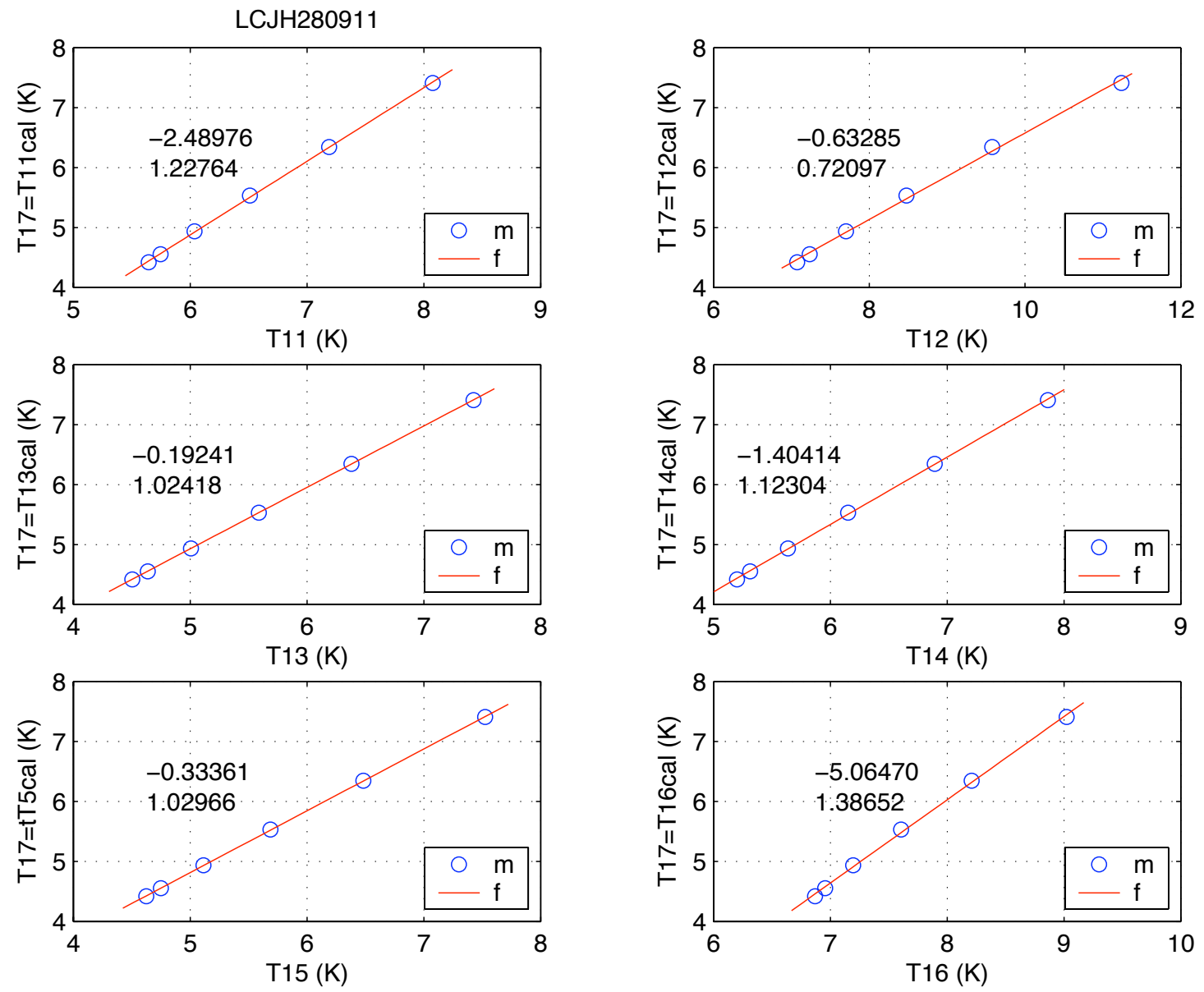

Figure 10: Calculation of the calibration parameters. Example in the sensor ring R1 of the run LCJH280911 (28 September 2005). In abscissa of each plot is the raw measurement (e.g. $T_{11}$, top left plot) and in ordinate the reference temperature in the central channel (e.g. $\left.T_{17}\right)$, which is also the resulting calibrated temperature (e.g. $\left.T_{11 \text { cal }}\right)$. The linear fit $Y=p_{1}+p_{2} X$ (symbol f) of the experimental data (symbol $\mathrm{m}$ ), as well as the parameters of the linear fit $p_{1}$ (top) and $p_{2}$ (bottom), are shown. 

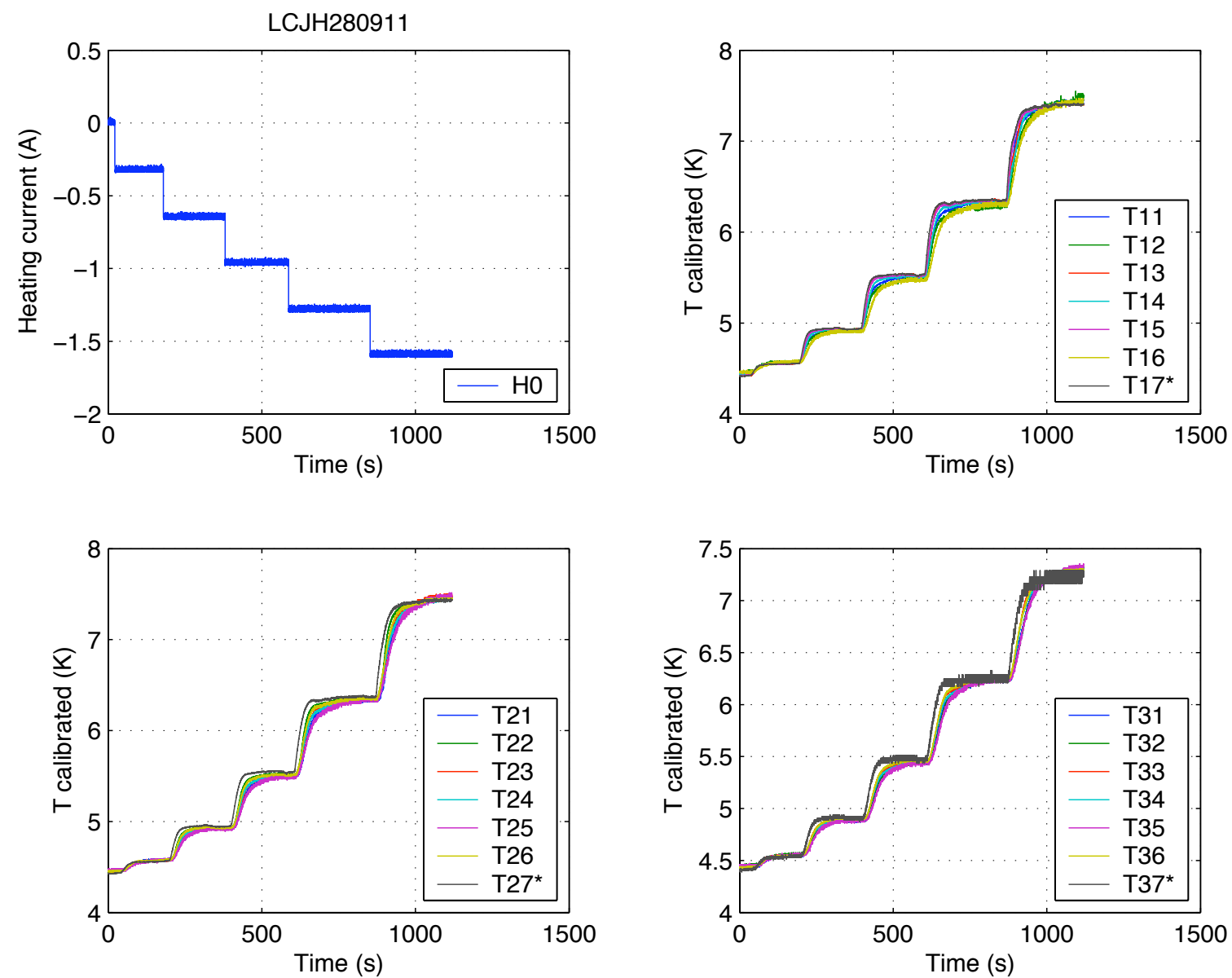

Figure 11: Result of the temperature calibration in the run LCJH280911 (28 September 2005). The time history of the heating current is shown in the upper-left plot. The time history of the calibrated temperatures at the rings R1, R2 and R3 are shown in the remaining three plots. The signal $T_{17} *, T_{27} *$ and $T_{37} *$ are the raw measurements. 

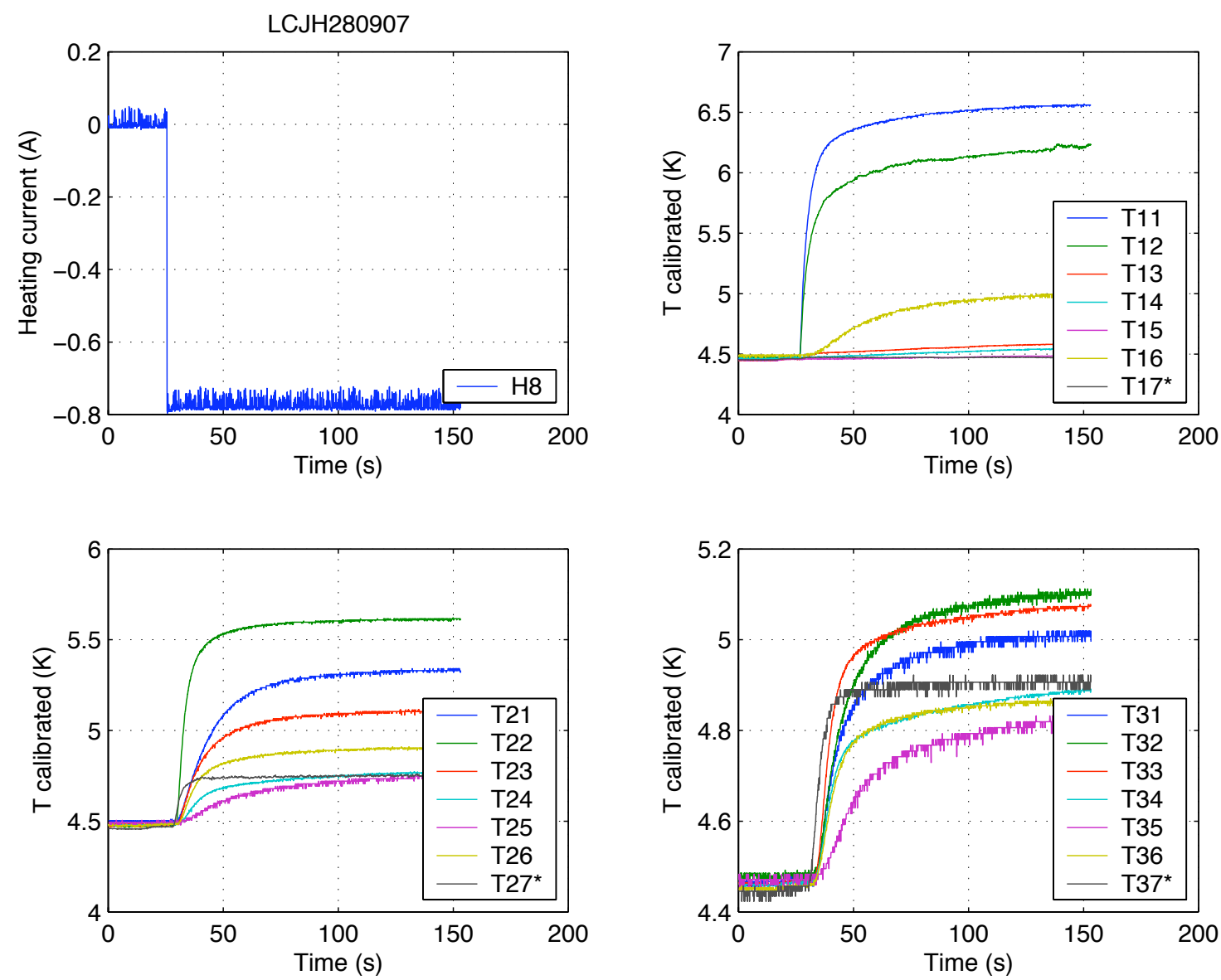

Figure 12: Result of calibration for the the typical run LCJH280907 ( $\dot{m}=8 \mathrm{~g} / \mathrm{s}$, heater H8), showing only the steady state part of the measurement (first heating step). The time history of the heating current is shown in the upper-left plot. The time history of the calibrated temperatures at the sensor rings R1, R2 and R3 are shown in the remaining three plots. The signal $T_{17} *, T_{27} *$ and $T_{37} *$ are the raw measurements. The error after re-scaling is $<50 \mathrm{mK}$. 


\section{Measurement analysis}

In a previous thermal hydraulic analysis of the ITER PFIS sample, also tested in SULTAN, we used the characteristic response time to assess $\mathrm{h}_{B H}$; the sample had only one sensor per ring and there was not enough information to calibrate the thermometers and quantify the offset at steady state [4]. Here the transfer heat transfer coefficients are assessed by a steady state analysis. This is possible because in the LCJ sample there are enough thermometers along the jacket circumference and along the sample axis so that they can be reliably calibrated, as discussed above.

The steady state temperature distribution along the azimuth angle $\phi$ is given by

$$
\Delta T_{i j}=T_{i j c a l}\left(t=t_{\text {end }}\right)-T_{i j c a l}(t=0)
$$

where the difference with the initial condition, rather than the steady state value, is used to eliminate the effect of the residual, albeit small, calibration error. The distribution $\Delta T_{i j}$ a periodic function of the angle $\phi$, as shown in the example (run LCJH280907) of Fig. 13.

\subsection{Fourier analysis}

As discussed in Appendix $\mathrm{A}$, the azimuthal temperature distribution $\Delta T_{i j}$ has several modes (eigenvectors) with associated characteristic length (eigenvalues). We apply the Fourier transform method to analyze the measurements (details are given in Appendix B):

$$
f_{i}=f_{F F T}\left(\Delta T_{i j}\right)
$$

The first Fourier coefficient $\left(A 1_{i}\right)$ corresponds to the average temperature variation in the bundle, and the module of the second coefficient $\left(a b s A 2_{i}\right)$ is proportional to the temperature difference among subcables in the bundle. For uniform heating the main mode excited will be a uniform temperature change in the cable, i.e. the first component of the Fourier transform. In the case of a localised heating (and in particular the heater H8), on the other hand, all modes are excited and we expect that the temperature distribution decays with a corresponding series of characteristic lengths. Given the analogy of eigenvectors and Fourier components discussed in Appendix A, we focus in the following analysis only on the first periodic component, i.e. the second coefficient of the Fourier transform, that shows a strong response in the runs analysed. This gives an additional benefit of filtering high frequency oscillations in the azimuthal temperature distribution, and thus providing a more stable quantity for the evaluation of the experimental results.

For the localized heater H8 (run LCJH280907), measurements and the reconstructed function

$$
\text { frec }_{i}=A 1_{i}+A 2_{i} \cos \phi-B 2_{i} \sin \phi
$$

are shown as a function of $\phi$ in Fig. 14. The modules of the Fourier coefficients, shown in the same Figure, have approximately the same order of magnitude at the sensor ring R1. This implies a strong non uniformity of temperature at this ring. This behavior is attenuated downstream in the axial direction due to heat exchange. 
Comparing the Fourier coefficients when using the other heaters. i.e. Fig. 15 (heater H0), Fig. $16(\mathrm{H} 3)$, Fig. $17(\mathrm{H} 7)$ and Fig. 18 (H9), at the same mass flow rate (e.g. 8g/s), the components of order $>1$ are negligible for all heaters except for the local heater H8. The temperature in the bundle is uniform; this confirms that the helium velocity in all subcables is approximately the same, as expected in a conductor without subcable wraps. A special case is represented by the helium heater $\mathrm{H} 7$ at the ring R1: here the first coefficient is negligible since the heat redistribute even in the short distance between heater and sensors $(70 \mathrm{~mm})$.

Qualitatively, all these results are in agreement the expected behavior. The dependence of the thermal hydraulic behavior on the mass flow rate is discussed below.

\subsection{Axial temperature distribution}

We analyse the following quantities along the sample axis:

- $A 1_{i}-\Delta T_{i 7}$, i.e. the difference between the average temperature variation in the bundle and in the central channel, where $\Delta T_{i 7}=T_{i 7}\left(t=t_{\text {end }}\right)-T_{i 7}(t=0)$, and

- $a b s A 2_{i}$.

A new coordinate system is used whose origin coincides with the location of each heater $(X *)$. In the special case of the heater $\mathrm{H} 0$ the origin is assumed to be at the sample inlet since the distance from this location to the actual heater is not relevant for the analysis.

At all mass flow rates the distribution of $A 1_{i}-\Delta T_{i 7}$ with $X *$ is flat when the heaters $\mathrm{H} 0$ and $\mathrm{H} 3$ are used (Fig. 19). The helium temperature in bundle and central channel is always uniform when using $\mathrm{H} 0$, and it becomes uniform when using $\mathrm{H} 3$ because the distance of $\approx$ $0.5 \mathrm{~m}$ is sufficient to redistribute the deposited heat. The variable $A 1_{i}-\Delta T_{i 7}$ decays along $X *$ in case of the heaters $\mathrm{H} 8$ and $\mathrm{H} 9$ since $T_{B}>T_{H}$, and increases for $\mathrm{H} 7$ since $T_{H}>T_{B}$. Finally, temperature difference and characteristic length are proportional to the increasing mass flow rate since the helium velocity also increases, as discussed below. The distribution along $X *$ of $a b s A 2_{i}$ is shown at all mass flow rates in Fig. 20. As already mentioned, only the local heater $\mathrm{H} 8$ has a non flat distribution in $X *$, and this is independent of the mass flow rate. Also in this case temperature difference and characteristic length are proportional with $\dot{m}$. All these qualitative results are in agreement with the expected results.

The exponents $k_{B H}$ and $k_{B B}$ are derived through exponential fits:

$$
\begin{aligned}
A 1_{i}-\Delta T_{i 7} & =a e^{-k_{H B} X *} \\
a b s A 2_{i} & =b e^{-k_{B B} X *}
\end{aligned}
$$

Their inverse values are the characteristic lengths $\Lambda_{B H}=1 / k_{B H}$ and $\Lambda_{B B}=1 / k_{B B}$. For the local heater $\mathrm{H} 8, \Lambda_{B H}$ varies in the range between $0.24 \mathrm{~m}$ at $2 \mathrm{~g} / \mathrm{s}$ and $0.47 \mathrm{~m}$ at $8 \mathrm{~g} / \mathrm{s}$, and $\Lambda_{B B}$ varies between $0.33 \mathrm{~m}(2 \mathrm{~g} / \mathrm{s})$ and $0.51 \mathrm{~m}(8 \mathrm{~g} / \mathrm{s})$. For the annular heater $\mathrm{H} 9$ the characteristic lengths are shorter than for $\mathrm{H} 8$, e.g. $\Lambda_{B H}$ varies between $0.17 \mathrm{~m}(2 \mathrm{~g} / \mathrm{s})$ and $0.32 \mathrm{~m}(8 \mathrm{~g} / \mathrm{s})$. Finally, for the heater in the central spiral H7 the characteristic lengths are: 
$\Lambda_{B H}$ varies between $0.26 \mathrm{~m}(2 \mathrm{~g} / \mathrm{s})$ and $0.38 \mathrm{~m}(8 \mathrm{~g} / \mathrm{s})$. All $k_{H B}$ and $k_{H B}$ results are listed in Table 3, and two detailed examples are shown in Fig. 21 and Fig. 22.

\subsection{Flow splitting}

In order to calculate the transversal heat transfer coefficients, the mass flow in the bundle $\dot{m}_{B}$ and in the hole $\dot{m}_{H}$ are needed. The measurements provide, however, only the total mass flow rate $\dot{m}$. Therefore, the flow splitting in the two channels is assessed using an iterative algorithm.

The friction factors coefficients in the bundle area (Katheder correlation [7]) and in the central channel (Showa spiral correlation [8]), are:

$$
\begin{aligned}
f_{\text {Katheder }} & =\left(1 / v f_{B}{ }^{0.72}\right)\left(0.051+19.5 / R e_{B}{ }^{0.88}\right) \\
f_{\text {Showa }} & =0.3024 R e_{H}^{-0.0707}
\end{aligned}
$$

where $R e_{B}$ and $R e_{H}$ are the respective Reynolds numbers. In this analysis we use a correction factor for the correlation of the bundle $f_{B}=0.7 f_{\text {Katheder, }}$ as assessed by pressure drop measurements of a similar dual channel CICC with no subcable wraps [9]. Due to the presence of heater (H7) and thermometers $\left(T_{17}, T_{27}\right.$ and $\left.T_{37}\right)$, the flow in the central channel is perturbed and the friction is larger than in the case without instrumentation. Therefore, we do not include the correction factor $(=0.5)$ suggested by measurements in the mentioned pressure drop experiment [10], i.e. in this analysis we use $f_{H}=f_{\text {Showa }}$. The uncertainty on the flow repartition due to the uncertainty on the friction factors is assessed in a sensitivity study discussed below.

At all investigated $\dot{m}$ the mass flow in the central channel is smaller than the mass flow in the bundle. The ratio $\dot{m}_{H} / \dot{m}_{B}$ is a function of $\dot{m}$, e.g. is 0.89 at $2 \mathrm{~g} / \mathrm{s}$ and 0.68 at $8 \mathrm{~g} / \mathrm{s}$. The flow velocity in the hole $v_{H}$ is $0.1 \mathrm{~m} / \mathrm{s}$ at $2 \mathrm{~g} / \mathrm{s}$ and $0.3 \mathrm{~m} / \mathrm{s}$ at $8 \mathrm{~g} / \mathrm{s}$ : the ratio $v_{H} / v_{B}$ is 4.5 at $2 \mathrm{~g} / \mathrm{s}$ and 3.5 at $8 \mathrm{~g} / \mathrm{s}$ (Fig. 23). 


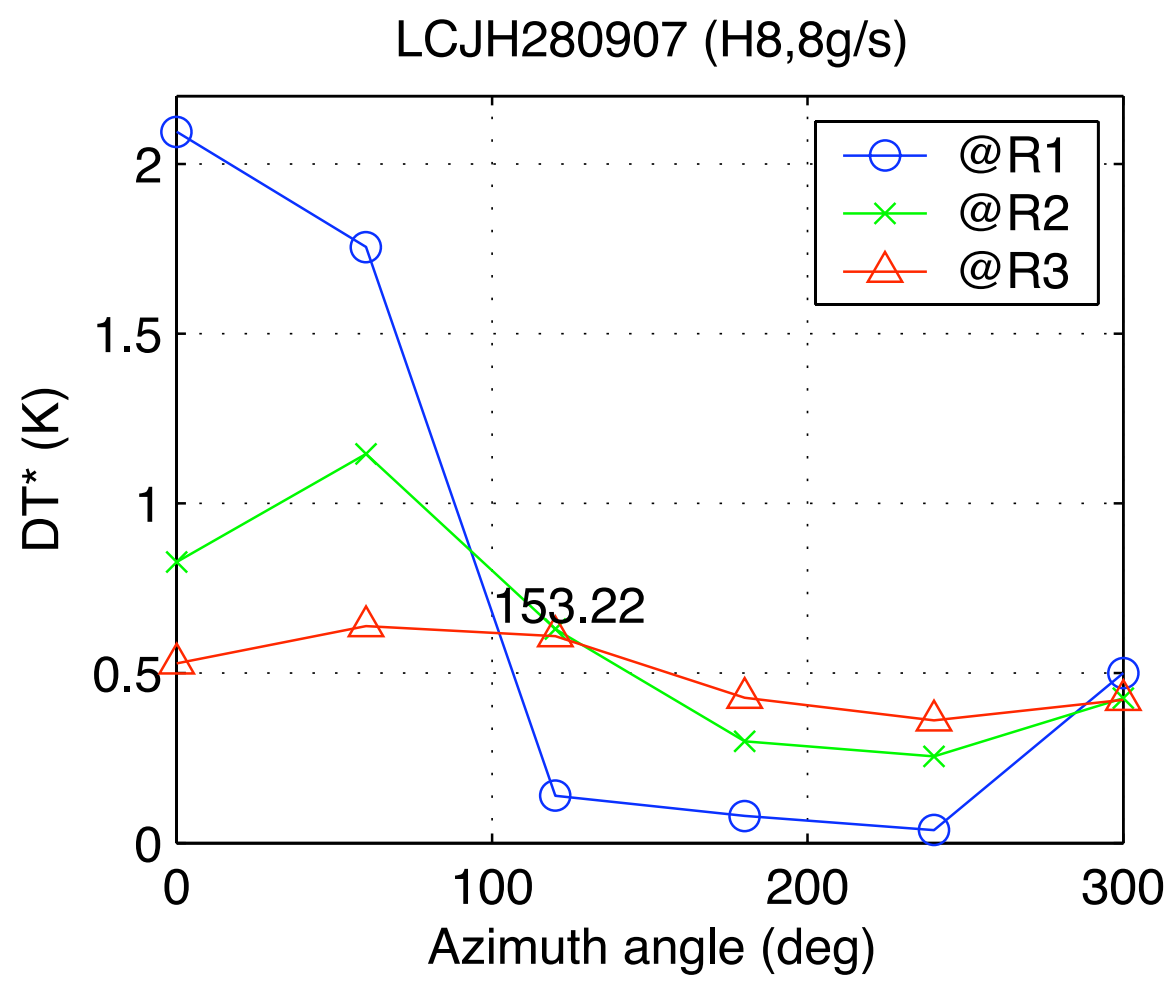

Figure 13: Results of run LCJH280907 ( $\dot{m}=8 \mathrm{~g} / \mathrm{s}$, heater H8) after the temperature calibration. The distribution of the calibrated temperatures (sensor ring R1, R2 and R3) is shown along the azimuth, i.e. the jacket circumference, at the end of the first heating step $t_{\text {end }}=153.22$ s. In particular, the quantity in ordinate $\left(\mathrm{DT}^{*}\right)$ is the difference between the calibrated temperature and its initial value. The temperatures in the central channel are: $T_{17}=0.0203 \mathrm{~K}, T_{27}=0.2979 \mathrm{~K}$ and $T_{37}=0.4607 \mathrm{~K}$ (not a function of the azimuth angle). 

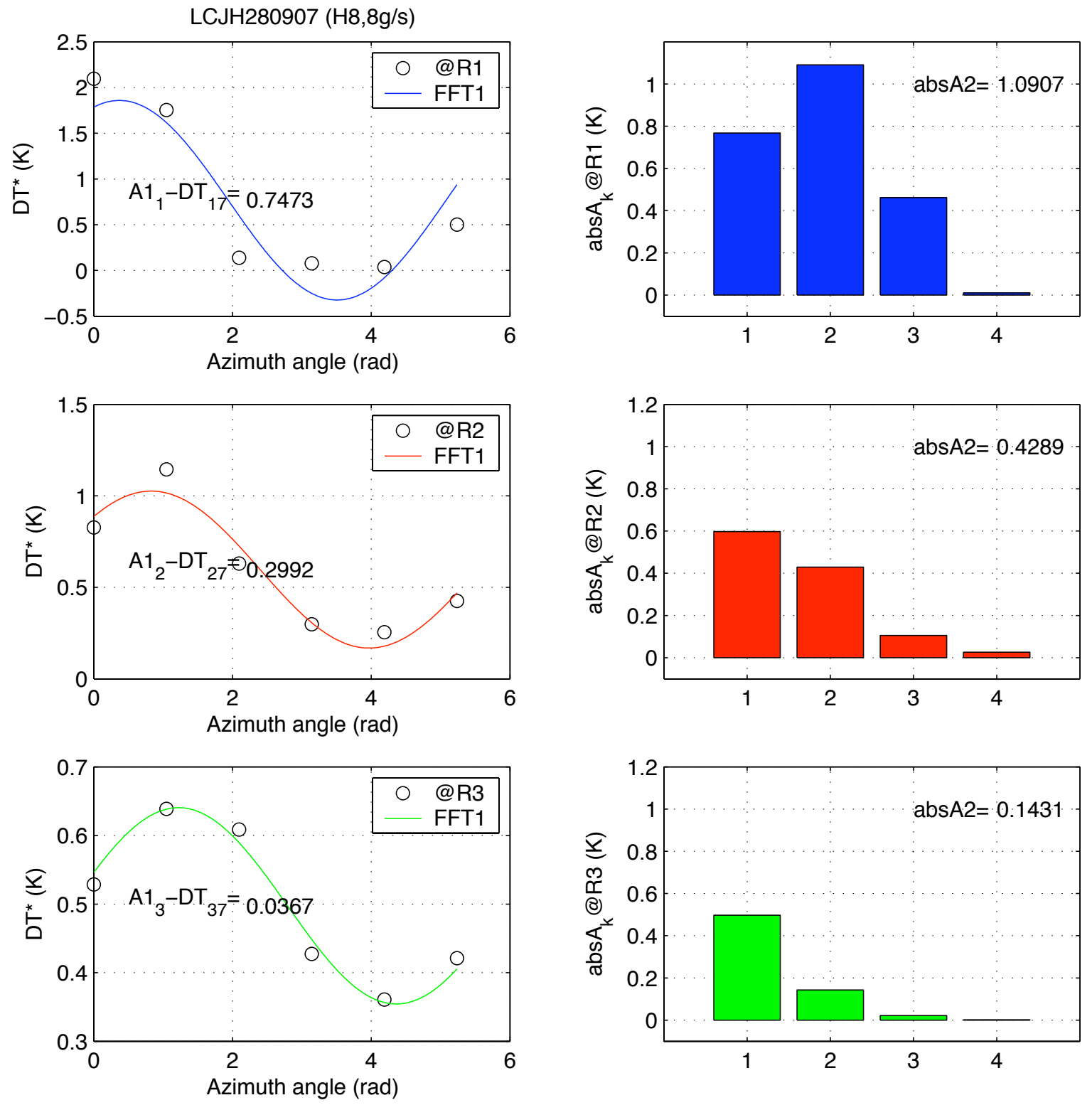

Figure 14: Results of run LCJH280907 ( $\dot{m}=8 \mathrm{~g} / \mathrm{s}$, heater H8) after application of the Fast Fourier Transform (FFT). All results are at $t_{\text {end }}=153.22 \mathrm{~s}$. Left plots: the distribution of the calibrated temperatures is shown along the azimuth, i.e. the jacket circumference. In particular, the quantity in ordinate (DT*) is the difference between the calibrated temperature and its initial value. The reconstructed function $\left(\mathrm{FFT1}=f r e c_{i}\right)$ is shown on the same plot. Right plots: the module of the coefficients of the FFT (see Appendix B). The results are shown at the sensor rings R1, R2 and R3. 

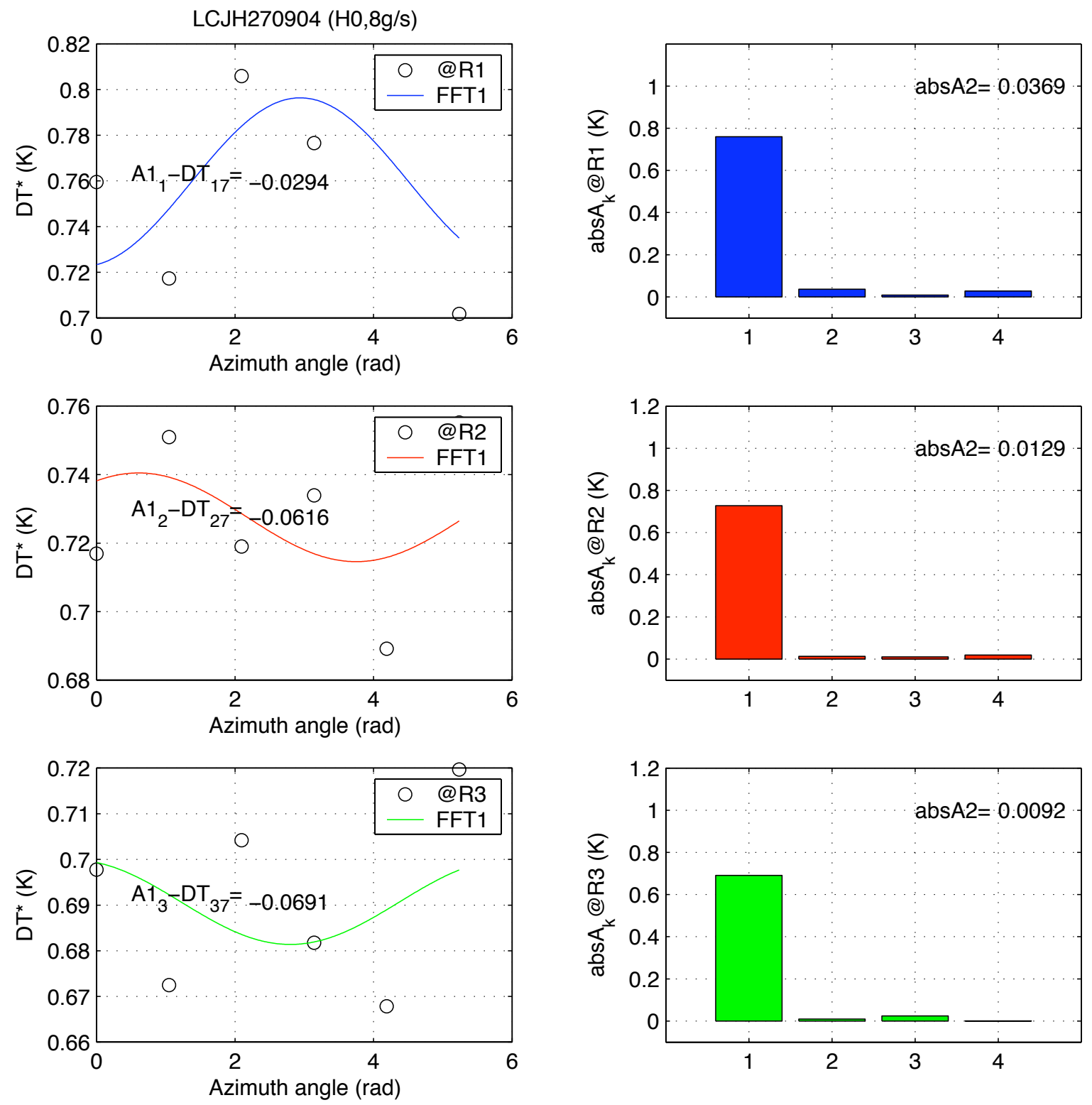

Figure 15: Results of run LCJH270904 ( $\dot{m}=8 \mathrm{~g} / \mathrm{s}$, heater H0) after application of the Fast Fourier Transform (FFT). All results are at $t_{e n d}=185 \mathrm{~s}$. The description of the plots is given in Fig. 14. 

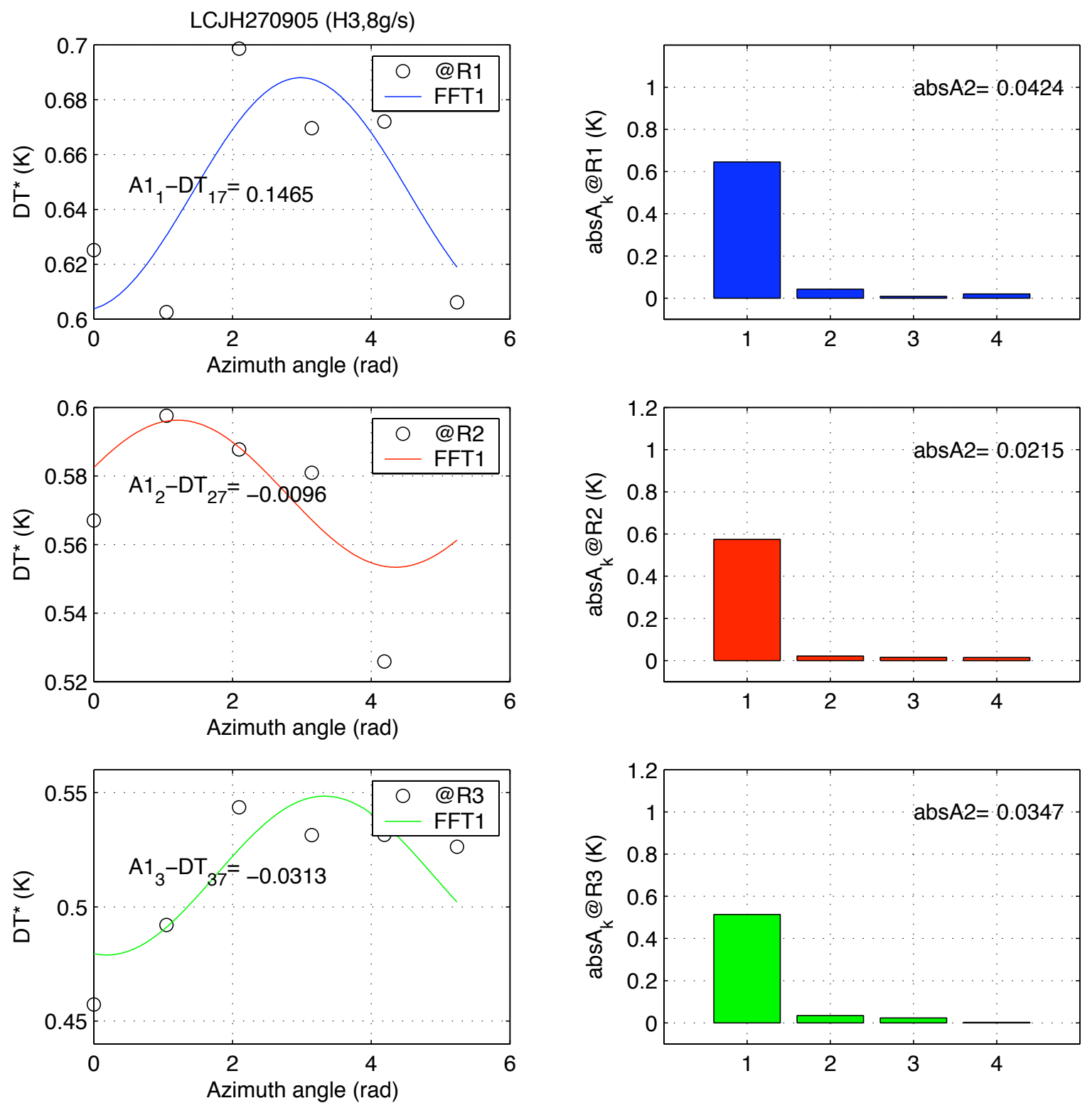

Figure 16: Results of run LCJH270905 ( $\dot{m}=8 \mathrm{~g} / \mathrm{s}$, heater H3) after application of the Fast Fourier Transform (FFT). All results are at $t_{\text {end }}=192 \mathrm{~s}$. The description of the plots is given in Fig. 14. 

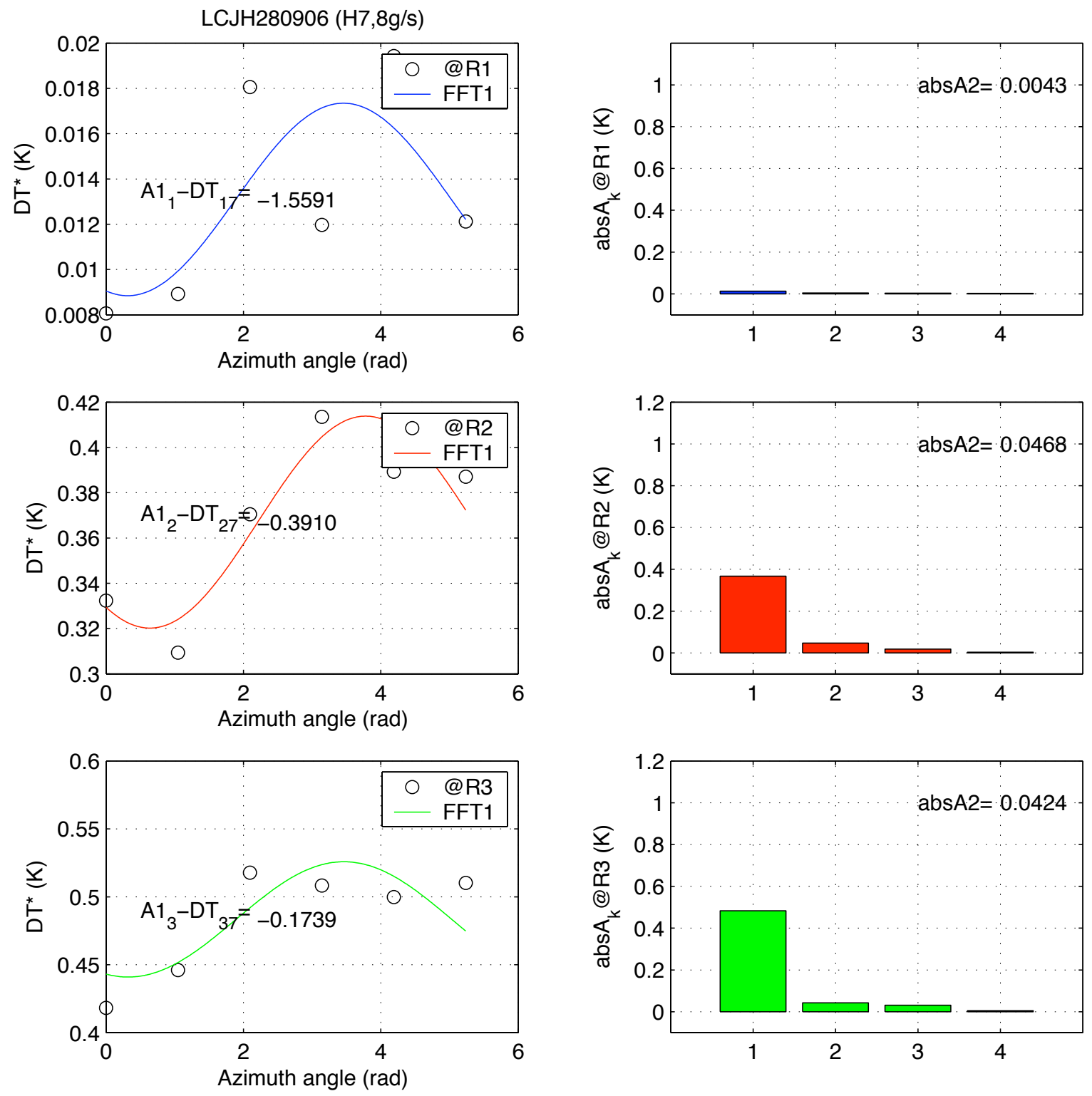

Figure 17: Results of run LCJH280906 ( $\dot{m}=8 \mathrm{~g} / \mathrm{s}$, heater H7) after application of the Fast Fourier Transform (FFT). All results are at $t_{\text {end }}=127 \mathrm{~s}$. The description of the plots is given in Fig. 14. 

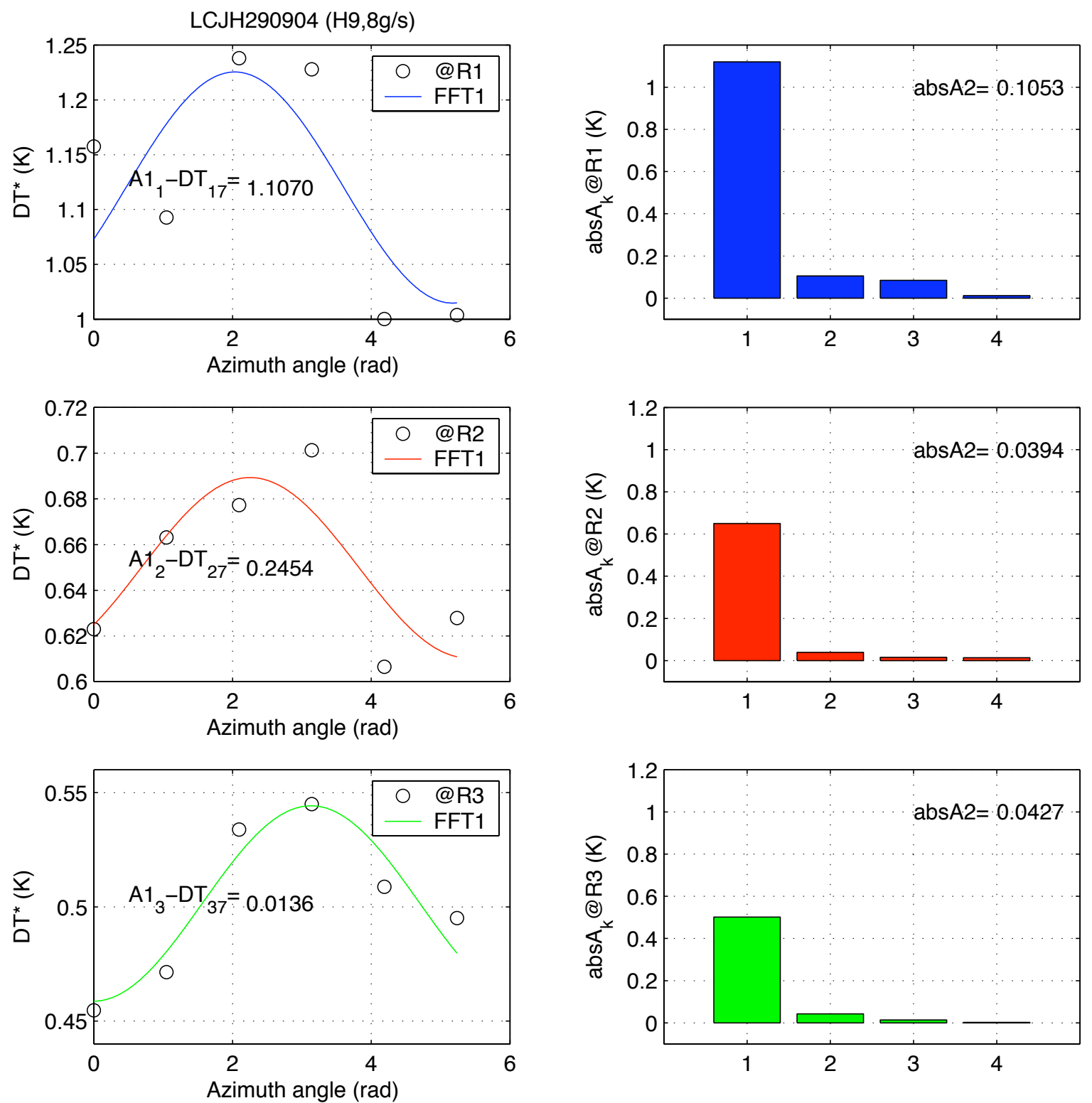

Figure 18: Results of run LCJH290904 ( $\dot{m}=8 \mathrm{~g} / \mathrm{s}$, heater H9) after application of the Fast Fourier Transform (FFT). All results are at $t_{\text {end }}=209 \mathrm{~s}$. The description of the plots is given in Fig. 14. 

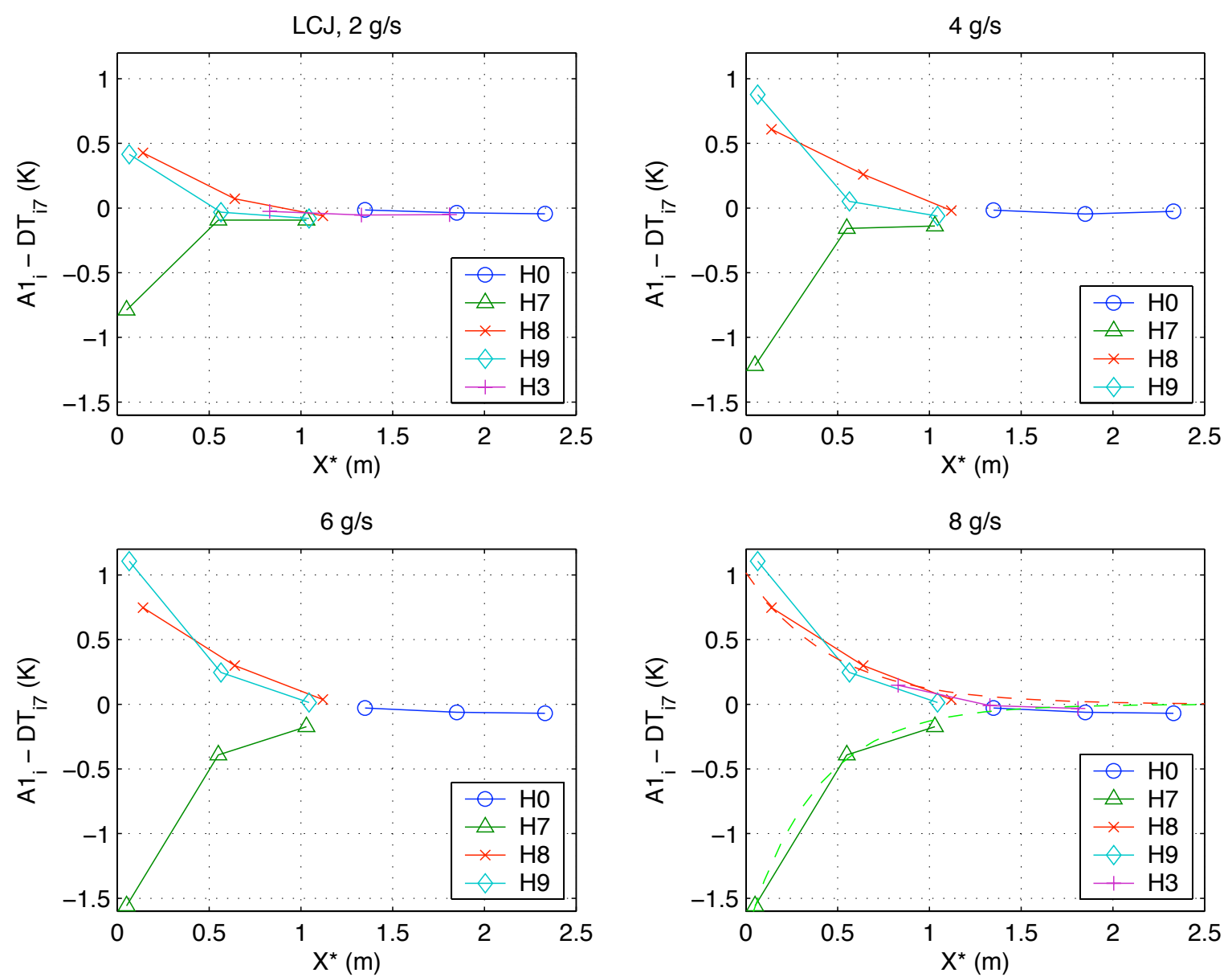

Figure 19: Summary of results of Fourier analysis. Axial temperature distribution $A 1_{i}-\Delta T_{i 7}$ along the coordinate $X *$, at all mass flow rates (e.g. on the top-left plot are the results at $2 \mathrm{~g} / \mathrm{s}$ ). The bottom-left plot shows also the exponential fit (dashed line) for the heater H8 and H7 (details in Fig. 21 and 22). The results of heater $\mathrm{H} 3$ at $4 \mathrm{~g} / \mathrm{s}$ and $6 \mathrm{~g} / \mathrm{s}$ are not consistent with the other results of the measurement set and therefore have been rejected in this report. 

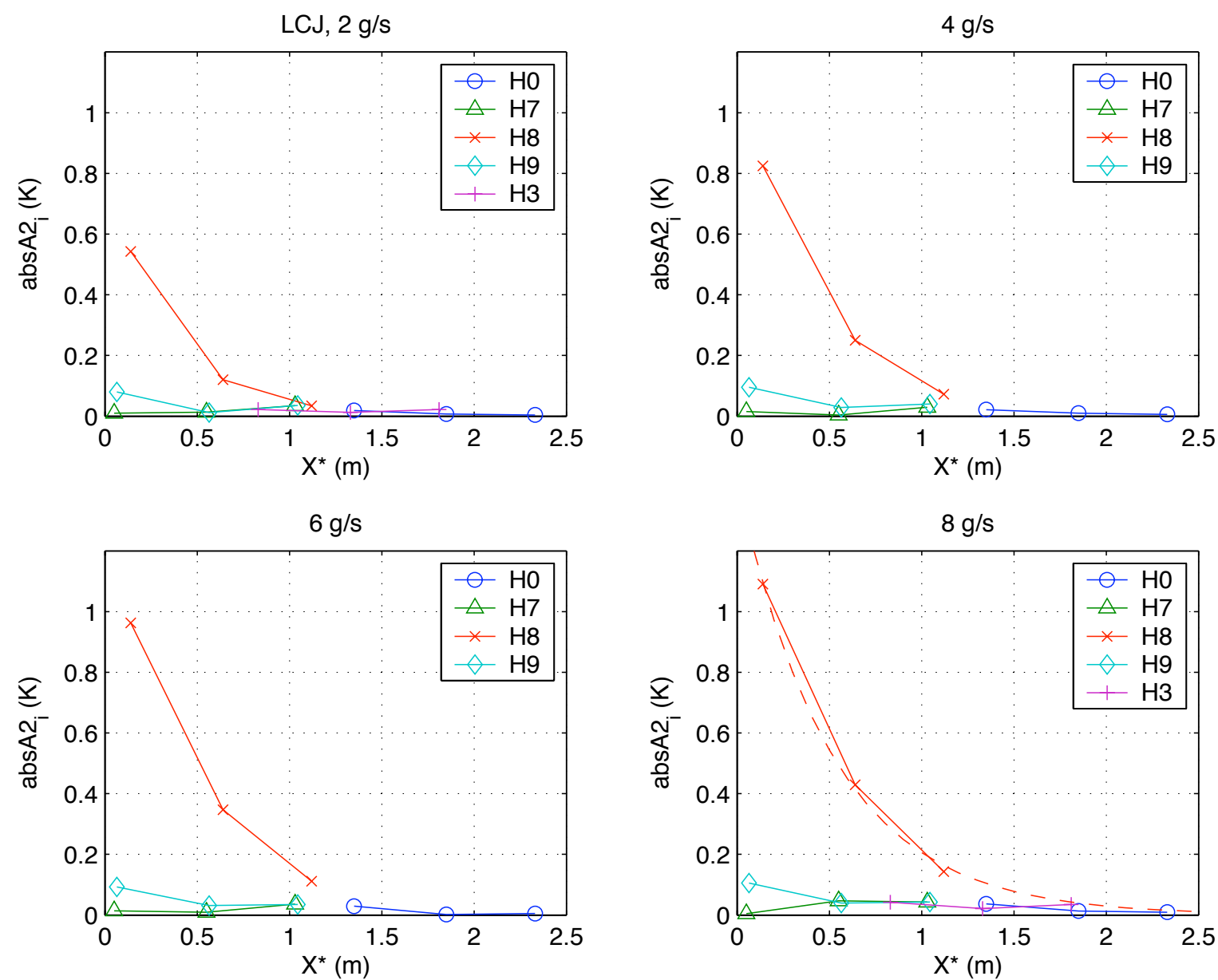

Figure 20: Summary of results of Fourier analysis. Axial temperature distribution $a b s A 2_{i}$ along the coordinate $X *$, at all mass flow rates (e.g. on the top-left plot are the results at $2 \mathrm{~g} / \mathrm{s}$ ). The bottom-left plot shows also the exponential fit (dashed line) for the heater H8 (details in Fig. 21). 

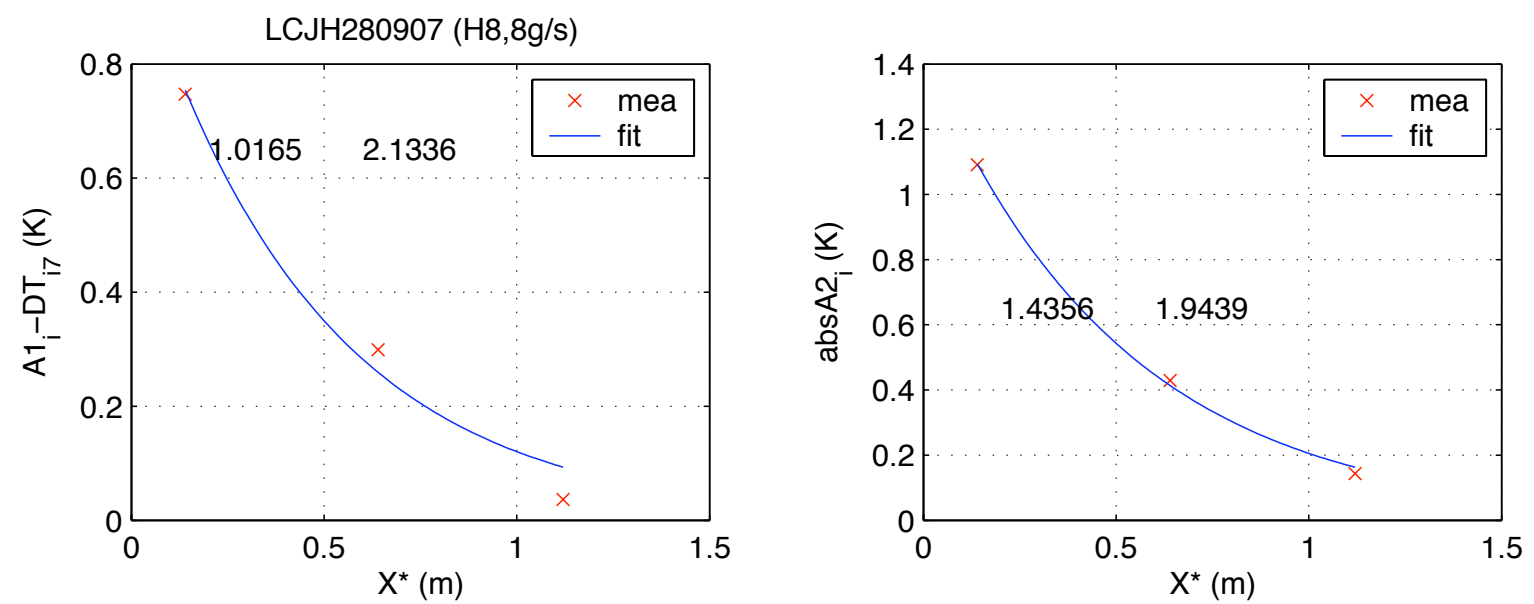

Figure 21: Example of exponential fit of the temperature distributions $A 1_{i}-\Delta T_{i 7}$ and $a b s A 2_{i}$ along the coordinate $X *$. Heater $\mathrm{H} 8$ at 8g/s (run LCJH280907). Left plot: measurement, fit and parameters $a$ (left) and $k_{B H}$ (right) are shown. Right plot: measurement, fit and parameters $b$ (left) and $k_{B B}$ (right) are shown.

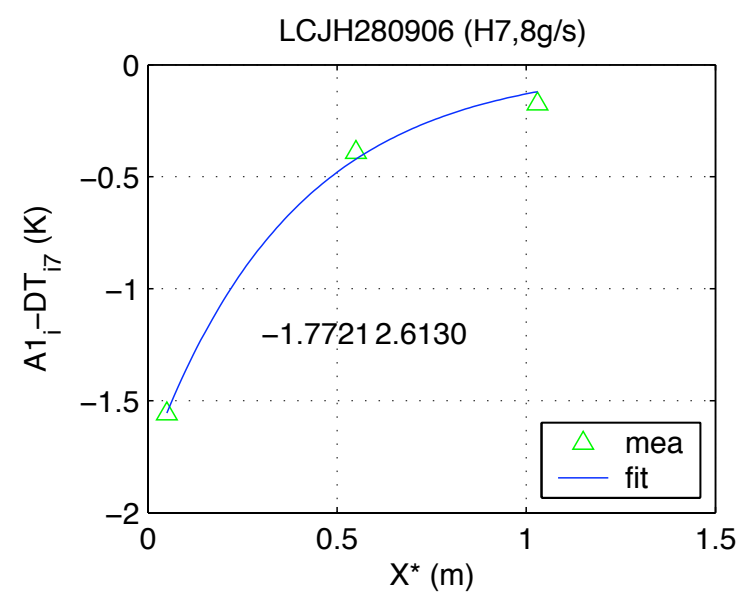

Figure 22: Example of exponential fit of the temperature distribution $A 1_{i}-\Delta T_{i 7}$ along the coordinate $X *$. Heater $\mathrm{H} 7$ at $8 \mathrm{~g} / \mathrm{s}$ (run LCJH280906). Measurements, fit and parameters $a$ (left) and $k_{B H}$ (right) are shown. 

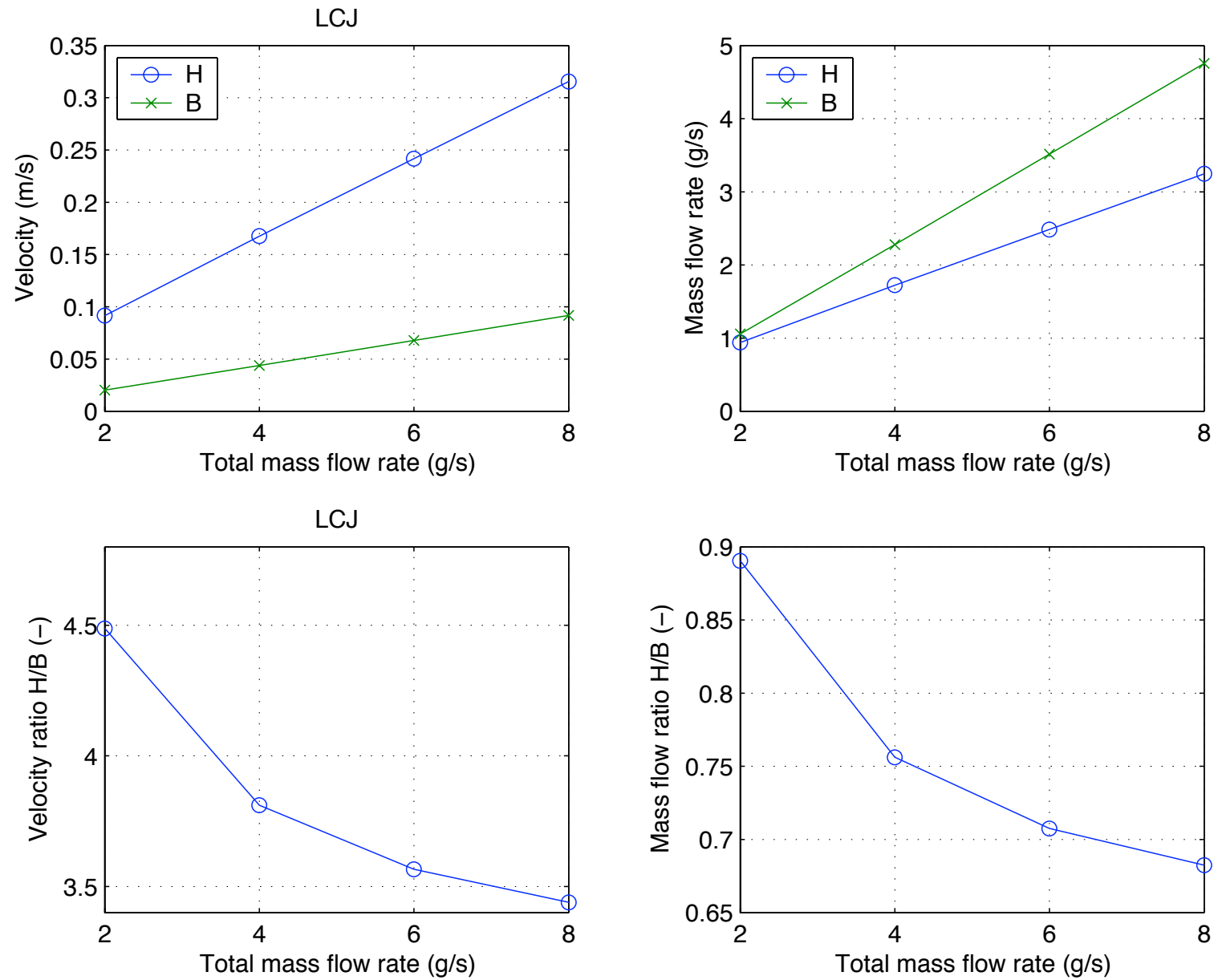

Figure 23: Results of flow splitting in the bundle (B) and hole (H) . Velocity and mass flow rate, as well as their ratios, are shown as a function of the total mass flow rate. The nominal friction factors are used, i.e. $f_{B}=0.7 f_{\text {Katheder }}$ and $f_{H}=f_{\text {Showa }}$. 


\section{Discussion}

In the experiment the temperature is measured in the center of the conductor (central hole), and at $N=6$ locations equally spaced in angle around the perifery. We model the heat transport assuming that this takes place in $N$ parallel cooling channels, located under the thermometers in the cable bundle, and in the central hole (Fig. 24). We assume steady state conditions, incompressible flow, negligible transverse flow, and constant thermophysical and transport properties. Under these assumptions the only relevant terms in the heat balances are longitudinal heat convection and mutual heat exchange. The balances can be written as a set of equations for the temperature in the parallel cooling channels. A detailed description of the method used here for the assessment of the azimuthal and radial heat transfer coeffients is given in Appendix A.

\subsection{Azimuthal heat transfer coefficient}

The azimuthal heat transfer coefficient between subcables in the bundle, derived from Eq. (73) in Appendix A, is

$$
h_{B B}=\frac{A_{B} \rho C p v_{B} k_{B B}}{2 p_{B B}}-\frac{p_{B H} h_{B H}}{2 p_{B B}}
$$

where $C_{p}$ is the helium specific heat at constant pressure, $\rho$ the helium density, $A_{B}=A_{B t o t} / 6$ is helium cross section of one subcable and $p_{B B}$ is the wetted perimeter between subcables, assumed to be $\left(d_{c s}-d_{o u t}\right) / 2$ (Table 1$)$.

The azimuthal coefficient $h_{B B}$ varies between $20 \mathrm{~W} / \mathrm{m}^{2} / \mathrm{K}$ at $2 \mathrm{~g} / \mathrm{s}$ to $120 \mathrm{~W} / \mathrm{m}^{2} / \mathrm{K}$ at $8 \mathrm{~g} / \mathrm{s}$. These values are considerably smaller than the radial coefficient $h_{B H}$, e.g. typically an order of magnitude at $2 \mathrm{~g} / \mathrm{s}$ and a factor 3 in the range $4-8 \mathrm{~g} / \mathrm{s}$ (Table 3 and Fig. 25). As discussed above, only the measurements of the local heater $\mathrm{H} 8$ can be used to asses $h_{B B}$.

\subsection{Radial heat transfer coefficient}

The radial heat transfer coefficient between bundle and central channel, derived from Eq. (72) in Appendix A, is

$$
h_{B H}=\frac{k_{B H}}{p_{B H}} \frac{1}{\frac{1}{A_{B} \rho C p v_{B}}+\frac{6}{A_{H} \rho C p v_{H}}}
$$

which is equivalent to

$$
h_{B H}=\frac{\dot{m}_{B} \dot{m}_{H} C_{p}}{\dot{m} p_{B H t o t} \Lambda_{B H}}
$$

as in [11]. $A_{H}$ is helium cross section of the central spiral, $p_{B H}=p_{B H t o t} / 6$ is the wetted perimeter between bundle and central channel for one subcable, and $p_{B H t o t}$ is assumed to be the outer circumference of the central spiral $(=35.81 \mathrm{~mm}$, Table 1$)$. 
The radial coefficient $h_{B H}$ varies between $200 \mathrm{~W} / \mathrm{m}^{2} / \mathrm{K}$ at $2 \mathrm{~g} / \mathrm{s}$ to $400-550 \mathrm{~W} / \mathrm{m}^{2} / \mathrm{K}$ at $8 \mathrm{~g} / \mathrm{s}$ and depends on the heater used (see Table 3 and Fig. 26). A comparison with the results of the ITER PFIS conductor is discussed below, at the end of the Section Interpretation.

\subsection{Sensitivity analysis}

A sensitivity analysis is performed to quantify the effect of the uncertainty on the friction factors on the transverse heat transfer coefficients. The range of the correction factors investigated is: $0.7-1.2$ for $f_{B}($ nominal value $=0.7)$ and $0.5-1.5$ for $f_{H}($ nominal value $=1.0)$.

- At the nominal $f_{H}$, the ratios $v_{H} / v_{B}$ and $\dot{m}_{H} / \dot{m}_{B}$ increase (quasi linearly proportional) with the increasing friction in the bundle. For example, in the range $4-8 \mathrm{~g} / \mathrm{s}$ the velocity ratio is 3 and the mass flow rate ratio is 0.6 at $f_{B}=0.5$, and the same ratios are 5 and 1 at $f_{B}=1.2$, respectively. The resulting radial coefficient $h_{B H}$ increases by $<10 \%$, and the azimuthal coefficient $h_{B B}$ decreases by $40 \%$ (Fig. 27).

- At the nominal $f_{B}$ and for increasing friction in the central spiral, the above results are qualitatively inversed, i.e. decrease of the radial coefficient by $<10 \%$ and an increase of azimuthal coefficient by almost a factor 2 (Fig. 28).

For a consistent use of the friction factor correlations the hydraulic diameter of the hole $D_{H}$ is assumed to be the outer diameter of the spiral. If $D_{H}$ is assumed to be the inner rather than the outer diameter of the spiral, $p_{B H t o t}=29.53 \mathrm{~mm}$ and $h_{B H}$ linearly increases by $\approx$ $20 \%$. The same change does not have an effect on the flow repartition between bundle and hole.

The error bars of $h_{B B}$ (Fig. 25) and $h_{B H}$ (Fig. 26) are indicative of the above uncertainties.

\subsection{Interpretation}

To interpret the results shown in Figs. 25 and 26 we use the results reported in Appendix C. As discussed there, the tortuosity of the helium flow results in an effective transverse thermal conductivity $k_{\perp}$ that can be significantly larger than that of stagnant helium. Given the value of thermal conductivity, that can be estimated using any of the three theories discussed in the Appendix, it is possible to compute an approximate value of the thermal resistance between bundle channels, and the corresponding heat transfer coefficient:

$$
h_{B B}=\frac{k_{\perp}}{d}
$$

where $d$ is the spacing between channels, which on average is:

$$
d \approx \frac{\pi}{12}\left(d_{c s}+D_{\text {out }}\right)
$$


The values of $h_{B B}$ resulting from the various approximations to $k_{\perp}$ discussed in the Appendix $C$ have a large scattering among the prediction of the various models. This is due to the large variability in the porous media for which the correlations have been established. Nonetheless, we see that there is a very good coherence between the measured results, increasing linearly with the massflow, and the expected linear dependency. In practice, we find that the empirical fit to the effective conductivity reported by Nield and Bejan [13] fits best the measured transverse heat transfer $h_{B B}$ when the coefficient $C_{T}=0.119$ (Fig. 25).

As for the heat transfer between the bundle channels and the cooling hole, we have applied a simple representation of heat transfer through the free-flow interface and through the spiral, as follows:

$$
h_{B H} \approx \frac{p_{\text {spiral }}}{\frac{1}{h_{B}^{\text {eff }}}+\frac{1}{h_{H}^{\text {eff }}}}+\frac{1-p_{\text {spiral }}}{\frac{1}{h_{B}^{\text {eff }}}+\frac{t_{\text {spiral }}}{k_{\text {spiral }}}+\frac{1}{h_{H}^{\text {eff }}}}
$$

where $p_{\text {spiral }}$ is the perforation of the spiral, $t_{\text {spiral }}$ its thickness and $k_{\text {spiral }}$ its thermal conductivity. The two heat transfer coefficients $h_{B}^{e f f}$ and $h_{H}^{e f f}$ are the effective heat transfer coefficient from the helium in the cable bundle and in the cooling hole at the hole-bundle interface. We estimate $h_{B}^{\text {eff }}$ using the Dittus-Boelter correlation corrected for the increased thermal conductivity of helium owing to thermal dispersion, i.e.:

$$
h_{B}^{e f f}=0.023 \operatorname{Re}_{B}^{0.8} \operatorname{Pr}^{1 / 3} \frac{k_{\perp}}{D_{B}}
$$

where $\operatorname{Pr}$ is the Prandtl number. For $h_{H}^{e f f}$ we use the results of Long [19] that demonstrated that the heat transfer coefficient in a pipe lined with a spiral is typically 2 to 5 times higher than that in a smooth pipe:

$$
h_{H}^{e f f}=h_{\text {extra }} 0.023 \operatorname{Re}_{H}^{0.8} \operatorname{Pr}^{1 / 3} \frac{k}{D_{H}}
$$

The results are reported in Fig. 26, demonstrating a reasonable agreement with the measured data using $h_{\text {extra }}$ in the range $2 . .5$, which is consistent with the experimental finding of Long.

The radial heat transfer coefficient $h_{B H}$ of the ITER PFIS conductor was assessed between $2 \mathrm{~g} / \mathrm{s}$ to $8 \mathrm{~g} / \mathrm{s}$ to be in the range $150-550 \mathrm{~W} / \mathrm{m}^{2} / \mathrm{K}$ in the conductor without subcable wraps, and $75-240 \mathrm{~W} / \mathrm{m}^{2} / \mathrm{K}$ in the conductor with subcable wraps [4]. These values are of the same order of magnitude of the results of the LCJ conductor. In fact, $h_{B H}$ is dominated by the presence of the central spiral, and the spirals of the PFIS and LCJ conductors are very similar, i.e. outer/inner diameter is $12 / 10 \mathrm{~mm}$ and $11.4 / 9.4 \mathrm{~mm}$, respectively, and the perforation is 0.25 for both. 
Table 3: Summary of results, i.e. exponent of Eq. (4) and Eq. (5) and transverse heat transfer coefficients in Eq. (8) and Eq. (10), using the nominal friction factors, i.e. $f_{B}=0.7 f_{\text {Katheder }}$ and $f_{H}=f_{\text {Showa }}$.

\begin{tabular}{cccccc}
\hline Heater & $\begin{array}{c}\dot{m} \\
(\mathrm{~g} / \mathrm{s})\end{array}$ & $\begin{array}{c}k_{H B} \\
(1 / \mathrm{m})\end{array}$ & $\begin{array}{c}k_{B B} \\
(1 / \mathrm{m})\end{array}$ & $\begin{array}{c}h_{H B} \\
\left(\mathrm{~W} / \mathrm{m}^{2} \mathrm{~K}\right)\end{array}$ & $\begin{array}{c}h_{B B} \\
\left(\mathrm{~W} / \mathrm{m}^{2} \mathrm{~K}\right)\end{array}$ \\
\hline H7 & 2 & 3.8318 & - & 175.24 & - \\
& 4 & 3.6747 & - & 330.76 & - \\
& 6 & 3.0550 & - & 408.23 & - \\
& 8 & 2.6130 & - & 462.55 & - \\
\hline H8 & 2 & 4.1754 & 2.9847 & 190.96 & 40.62 \\
& 4 & 2.2417 & 2.4128 & 201.77 & 119.16 \\
& 6 & 2.1467 & 2.0954 & 286.86 & 214.25 \\
& 8 & 2.1334 & 1.9439 & 377.65 & 313.97 \\
\hline H9 & 2 & 4.2958 & - & 196.47 & - \\
& 4 & 3.9421 & - & 354.83 & - \\
& 6 & 3.6002 & - & 481.09 & - \\
& 8 & 3.1501 & - & 557.63 & - \\
\hline
\end{tabular}




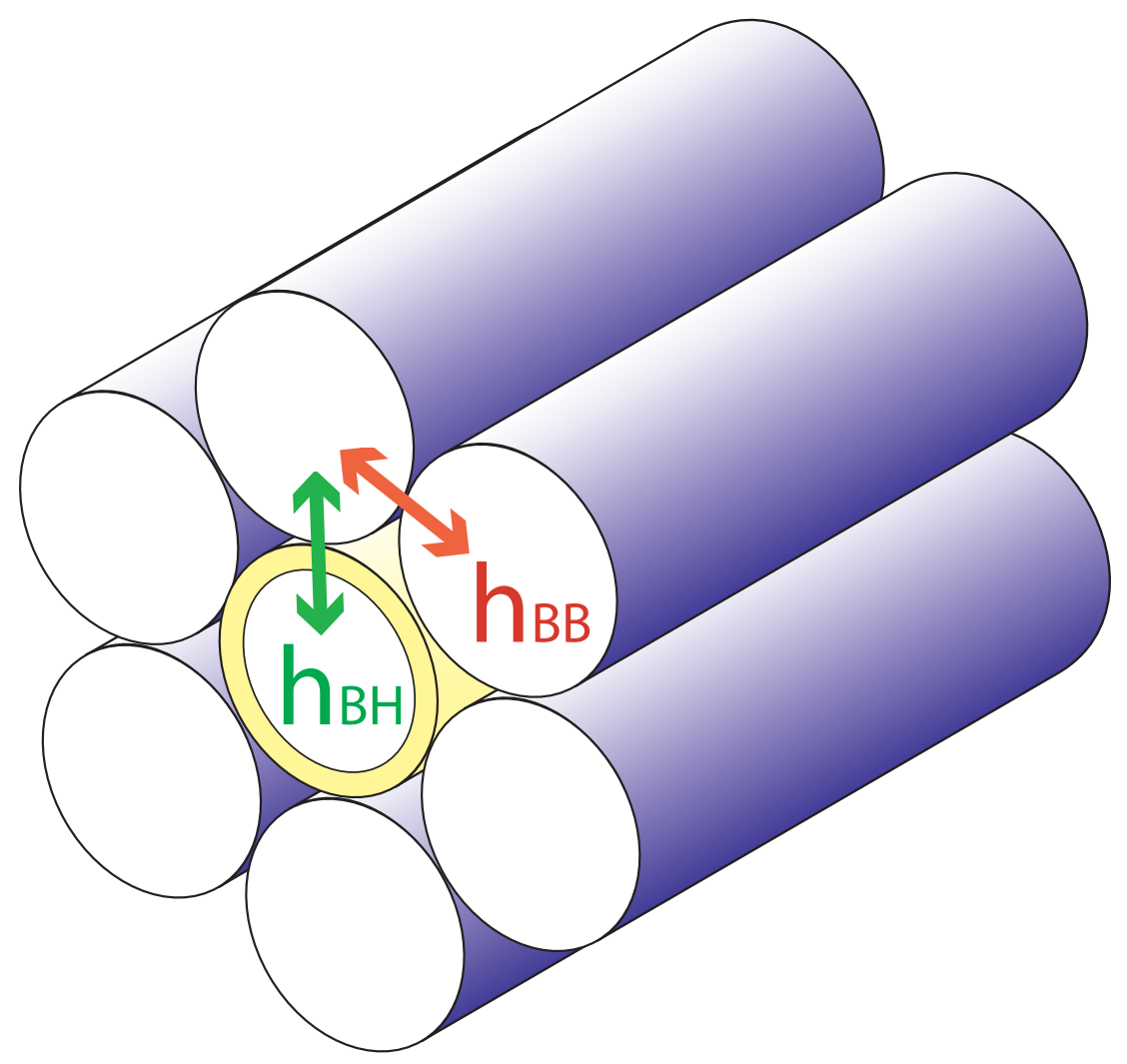

Figure 24: Schematic model of the conductor with central channel (hole) and $\mathrm{N}=6$ parallel cooling channels (bundle). The radial heat transfer coefficient between bundle and hole is $h_{B H}$, and the azimuthal heat transfer coefficient between subcables in the bundle is $h_{B B}$. 


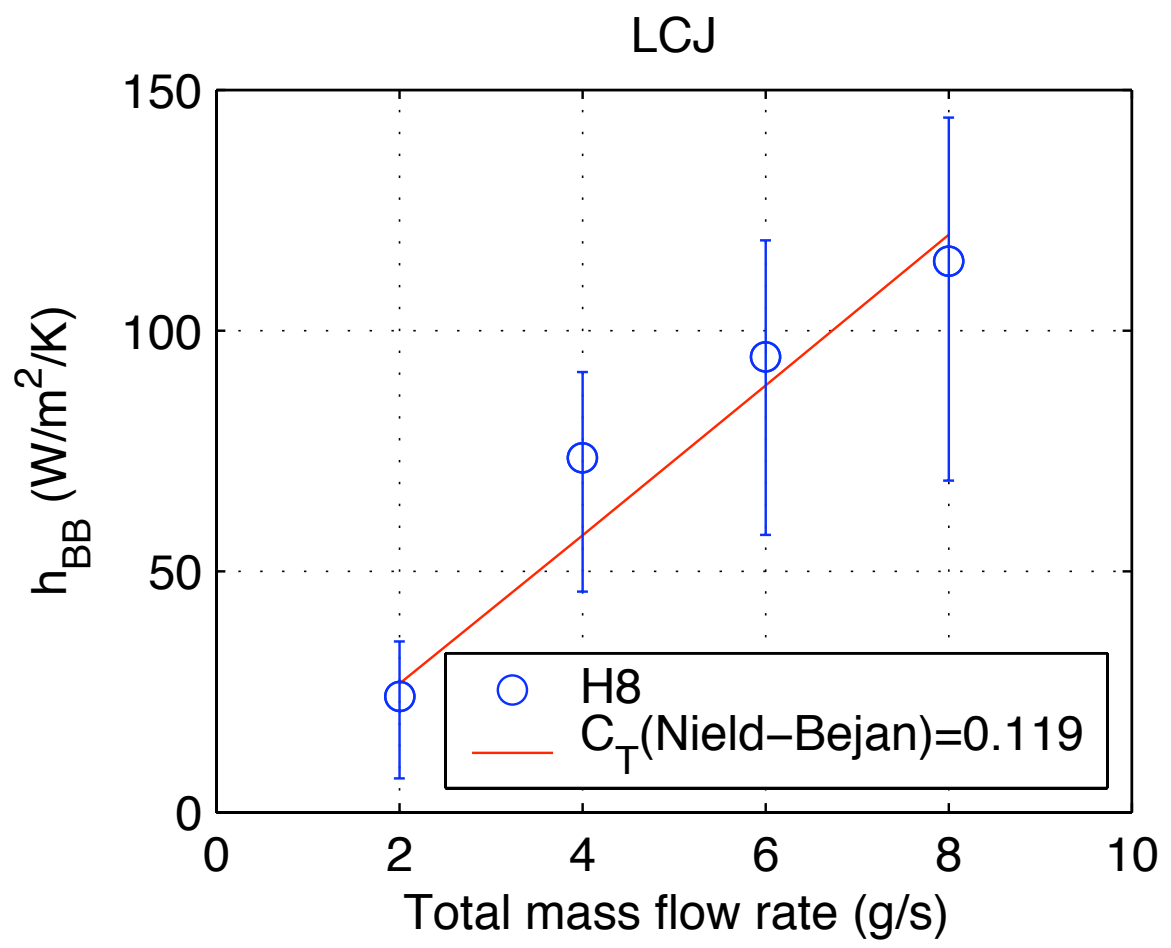

Figure 25: Azimuthal heat transfer coefficient between subcables in the bundle, as a function of the total mass flow rate (heater H8). The nominal friction factors are used, i.e. $f_{B}=0.7 f_{\text {Katheder }}$ and $f_{H}=f_{\text {Showa }}$. The fit of the experimental data using the Nield-Bejan expression with the coefficient $C_{T}=0.119$ is also shown (see Eq. 97 in Appendix C). 


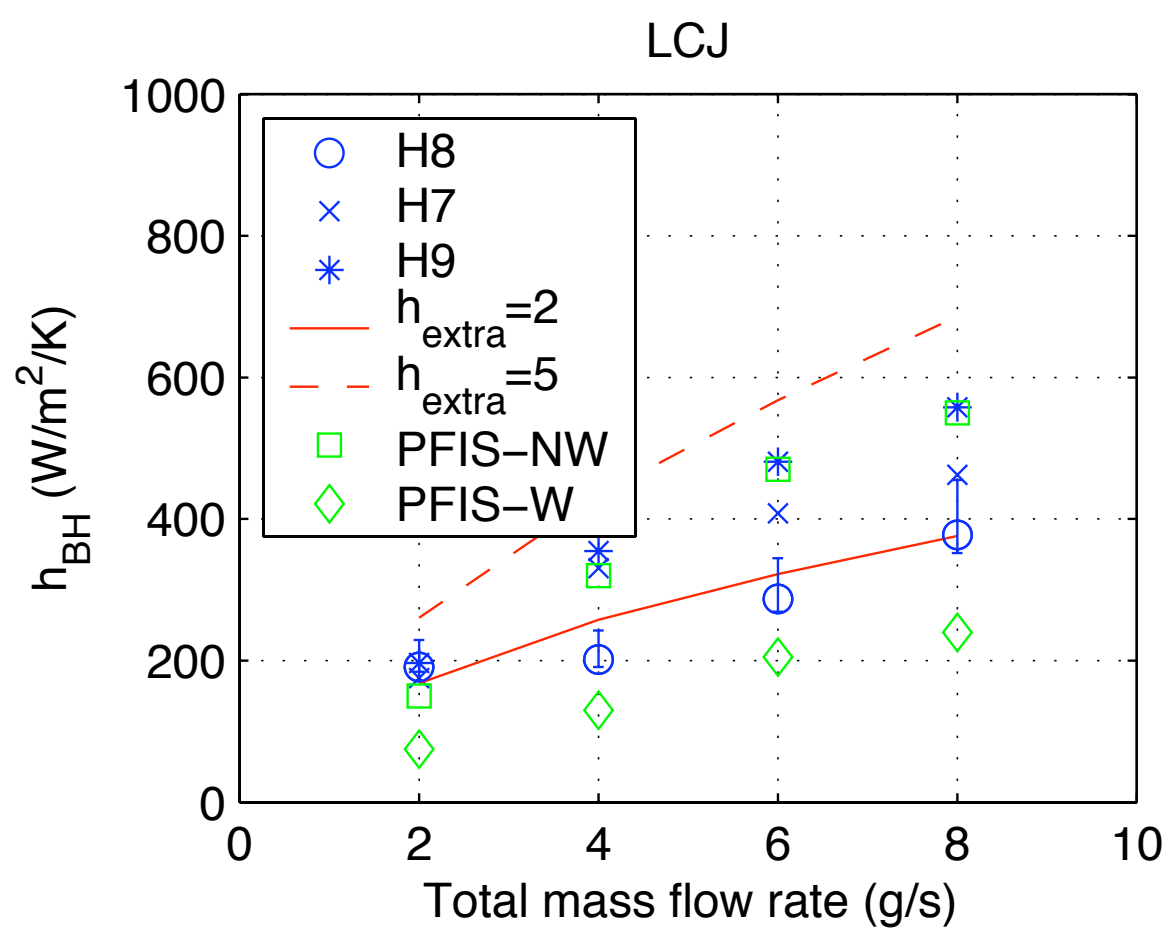

Figure 26: Radial heat transfer coefficient between bundle and hole, as a function of the total mass flow rate (heater H7, H8 and H9). The nominal friction factors are used, i.e. $f_{B}=0.7 f_{\text {Katheder }}$ and $f_{H}=f_{\text {Showa }}$. The results of the Long expression are also shown in the range of $h_{\text {extra }}$ between 2 and 5 (see Eq. 16). The results of the PFIS conductor are also included for comparison (PFIS-NW without subcable wraps, PFIS-W with subcable wraps) [4]. 

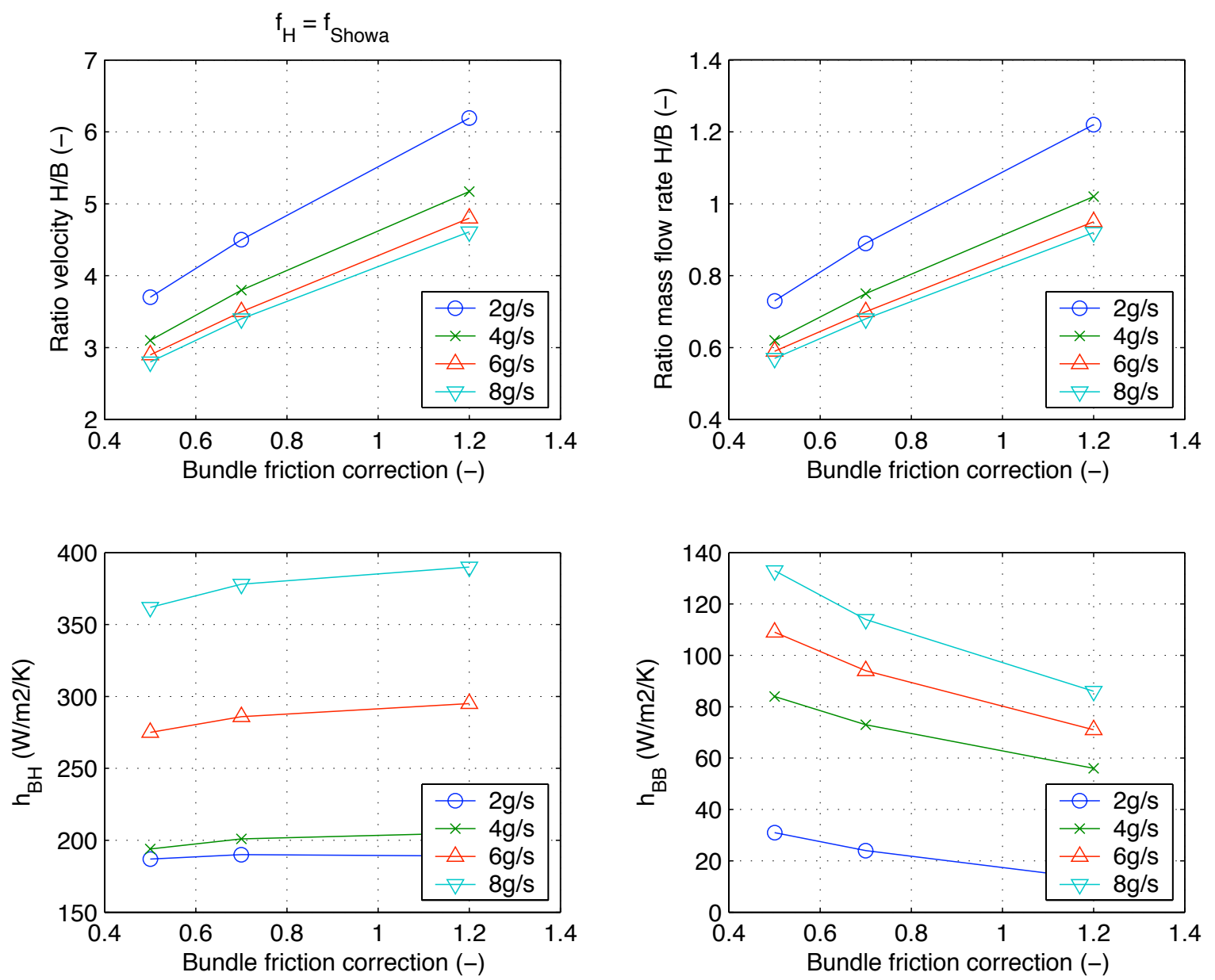

Figure 27: Results of the sensitivity analysis. The nominal friction factor in the central spiral is used $\left(f_{H}=f_{\text {Showa }}\right)$. The ratio of velocity $v_{H} / v_{B}$, the ratio of mass flow rate $\dot{m}_{H} / \dot{m}_{B}$, the radial heat transfer coefficient $h_{B H}$ and the azimuthal heat transfer coefficient $h_{B B}$ are plotted as a function of the correction of the friction factor in the bundle. The heating is provided by the heater $\mathrm{H} 8$. 

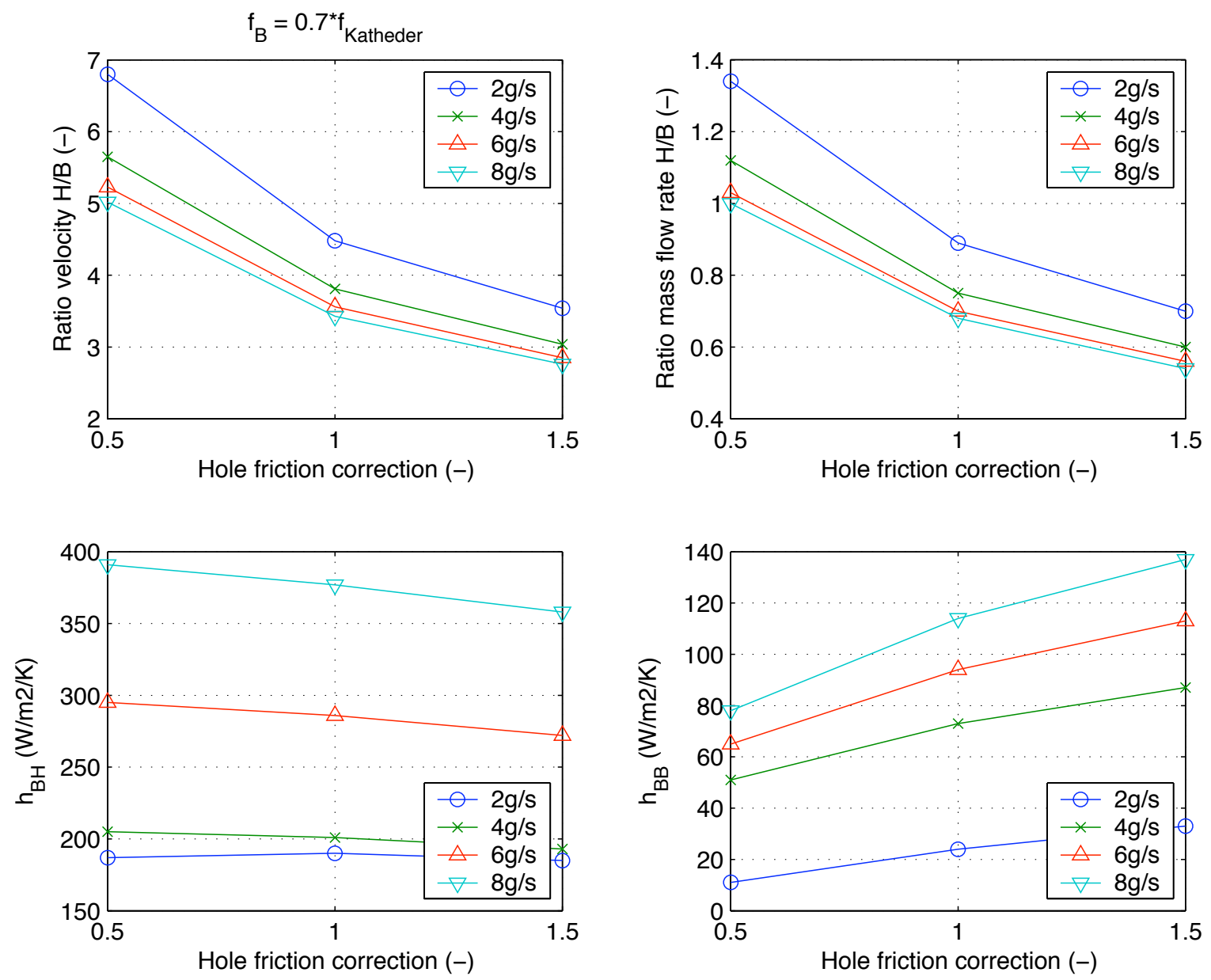

Figure 28: Results of the sensitivity analysis. The nominal friction factor in the bundle is used $\left(f_{B}=0.7 f_{\text {Katheder }}\right)$. The ratio of velocity $v_{H} / v_{B}$, the ratio of mass flow rate $\dot{m}_{H} / \dot{m}_{B}$, the radial heat transfer coefficient $h_{B H}$ and the azimuthal heat transfer coefficient $h_{B B}$ are plotted as a function of the correction of the friction factor in the hole. The heating is provided by the heater $\mathrm{H} 8$. 


\section{Conclusions}

An ITER-type dual channel CICC, referred to as the "Low Cost Joint" conductor, has been tested in SULTAN in 2005 with the main goal to asses its transverse heat transfer coefficients, namely the azimuthal heat transfer coefficient between subcables in the bundle, and the radial heat transfer coefficient between bundle and central channel. The main conclusions of the study which has focused on this experiment are the following:

- The special instrumentation of the sample has allowed the precise measurement of the local temperatures as well as the calibration to obtain reliable steady state values.

- The analytical solution for heat exchange among parallel pipes in a system with an arbitrary number of channels has allowed to derive the transverse heat transfer coefficients.

- The results can be explained if the CICC is considered as a porous medium. This hypothesis is consistent with previous findings, e.g. Katheder and Long.

- The applied method provides a simple and powerful means to extrapolate and predict transverse heat transfer coefficients of cables.

This study integrates and concludes the work started with the ITER PFIS conductor [?].

\section{Acknowledgments}

It is a real pleasure to thank Valeri Markushin (PSI) for many fruitful discussions about the measurement analysis, the numerical solution, and the $\mathrm{AT}_{\mathrm{E}} \mathrm{X}$ documentation, and Walter Venturini (CERN) for discussions on the analytical solution.

First compiled: 21st February 2006

Last modified: 20th September 2006

Typeset by ${ }^{A T} T_{E} X$ 


\section{References}

[1] "Final Report on PF Joint Sample", CRPP Rep. EFDA GB8 M29 Del. 3b (ITER Task N11TT03), 2004.

[2] B. Stepanov, P. Bruzzone, M. Vogel, "A low cost joint for the ITER PF coils, design and test results ", Fusion Engineering and Design, 75-79, 259-263, 2005.

[3] P. Bruzzone, B. Stepanov, M. Vogel, "A thermal hydraulic experiment on dual channel CICC to investigate local heat transfer", to be published in Cryogenics.

[4] L. Bottura, P. Bruzzone, C. Marinucci, B. Stepanov, "Analysis of transverse heat transfer coefficient in CICC's with central cooling channel", Cryogenics, 46, 597-605, 2006

[5] C. Marinucci, L. Bottura, P. Bruzzone, B. Stepanov, "Analysis of the transverse heat transfer coefficient in a dual channel ITER-type cable-in-conduit conductor", Presented at CHATS-AS 2006 and submitted for publication in Cryogenics

[6] L. Bottura, C. Marinucci, "Thermal dispersion in CICC's", Presented at CHATS-AS 2006 and submitted for publication in International Journal of Heat and Mass Transfer

[7] H. Katheder "Optimum thermohydraulic operation regime for cable-in-conduit superconductors (CICS", Cryogenics, 34, 595-598, 1994.

[8] S. Nicollet, J.L. Duchateau, H. Fillunger, A. Martinez, S. Parodi, "Ducal channel cablein-conduit thermohydraulics: influence of some design parameters", IEEE Trans. on Appl. Superc., 10, 1102-1105, 2000.

[9] C. Marinucci, P. Bruzzone, A. della Corte, L. Savoldi, Richard, R. Zanino, "Pressure drop of the ITER PFCI cable-in-conduit conductor", IEEE Trans. Appl. Supercond., 15, 1383-1386, 2005.

[10] C. Marinucci, "Pressure drop of the ITER PFIS cable-in-conduit conductor. Second measurement campaign: Hole open (2005)", CRPP Report: CRPP/SC/CM/2005/01, 2005.

[11] B. Renard, J.L. Duchateau, B. Rousset, L. Tadrist, "Evaluation of thermal gradients and thermosiphon in dual channel cable-in-conduit conductors", Cryogenics, 46, 530-540, 2006

[12] T. Harman, J. Dabney, N. Richert, "Advanced engineering mathematics using Matlab", PWS Publishing Company, ISBN 0-534-94350-0, 1997.

[13] D.A. Nield, A. Bejan, "Convection in porous media", Springer-Verlag, New York, 1992.

[14] C.T. Hsu, P. Cheng," Thermal dispersion in a porous medium", Int. J. Heat Mass Transfer, 33(8), 1587-1597, 1990.

[15] K.K. Kar, A. Dybbs, "Internal heat transfer coefficients of porous metals", Heat Transfer in Porous Media, J.V. Beck and L.S. Yao Editors, HTD-22, ASME, 1982.

[16] Y. Bo-Ming, L. Jian-Hua, "A fractal model for the transverse thermal dispersion conductivity in porous media", Chin. Phys. Lett., 21(1), 117-120, 2004. 
[17] R.B. Bird, W.E. Stewart, E.N. Lightfoot, "Transport phenomena", John Wiley \& Sons, 2002.

[18] J.C. Ward, "Turbulent flow in porous media", ASCE J. Hydraul. Div., 90(HY5), 1-12, 1964.

[19] A.E. Long, "Transverse heat transfer in a cable-in-conduit conductor with central cooling channel", M. Sc. Thesis, MIT, 1995. 


\section{Appendix A}

\section{Analytical solution}

In the experiment the temperature is measured in the center of the conductor (central hole), and at $N$ locations equally spaced in angle around the perifery. We model the heat transport assuming that this takes place in $N$ parallel cooling channels, located under the thermometers in the cable bundle, and in the central hole. We assume steady state conditions, incompressible flow, negligible transverse flow, and constant thermophysical and transport properties. Under these assumptions the only relevant terms in the heat balances are longitudinal heat convection and mutual heat exchange. The balances can be written as a set of equations for the temperature in the parallel cooling channels:

$$
A_{B} \rho C_{p} v_{B} \frac{\partial T_{i}}{\partial x}=p_{B B} h_{B B}\left(T_{i-1}-T_{i}\right)+p_{B B} h_{B B}\left(T_{i+1}-T_{i}\right)+p_{B H} h_{B H}\left(T_{H}-T_{i}\right)
$$

for $i=1 \ldots N$, and

$$
A_{H} \rho C_{p} v_{H} \frac{\partial T_{H}}{\partial x}=\sum_{i=1}^{N} p_{B H} h_{B H}\left(T_{i}-T_{H}\right)
$$

$C_{p}$ is the helium specific heat at constant pressure, $\rho$ the helium density, $A_{B}$ and $A_{H}$ are the helium cross section in bundle and hole, $v_{B}$ and $v_{H}$ are the helium velocity in bundle and hole, $p_{B B}$ is the wetted perimeter between subcables in the bundle, $p_{B H}$ is the wetted perimeter between bundle and hole for one subcable, $h_{B B}$ is the azimuthal heat transfer coefficient between subcables in the bundle and $h_{B H}$ is the radial heat transfer coefficient between bundle and hole, $T_{i}$ is the temperature in the $i t h$ subcable, $T_{H}$ is the hole temperature, and $x$ is the coordinate along the pipes.

The boundary conditions are of a temperature step in the bundle cooling channel under a thermometer, and uniform temperature everywhere else:

$$
\begin{gathered}
T_{i}(0)=\left\{\begin{array}{cc}
T_{0}+\Delta T & \text { for } i=1 \\
T_{0} & \text { for } 2 \leq i \leq N
\end{array}\right. \\
T_{H}(0)=T_{0}
\end{gathered}
$$

To arrive at an analytical solution, we write the equation as follows:

$$
\frac{\partial T_{i}}{\partial x}=\frac{p_{B B} h_{B B}}{A_{B} \rho C_{p} v_{B}}\left(T_{i-1}-T_{i}\right)+\frac{p_{B B} h_{B B}}{A_{B} \rho C_{p} v_{B}}\left(T_{i+1}-T_{i}\right)+\frac{p_{B H} h_{B H}}{A_{B} \rho C_{p} v_{B}}\left(T_{H}-T_{i}\right)
$$


for $i=1 \ldots N$, and

$$
\frac{\partial T_{H}}{\partial x}=\sum_{i=1}^{N} \frac{p_{B H} h_{B H}}{A_{H} \rho C_{p} v_{H}}\left(T_{i}-T_{H}\right)
$$

We introduce the parameters:

$$
\begin{aligned}
\alpha & =\frac{p_{B B} h_{B B}}{A_{B} \rho C_{p} v_{B}} \\
\beta & =\frac{p_{B H} h_{B H}}{A_{B} \rho C_{p} v_{B}} \\
\gamma & =\frac{p_{B H} h_{B H}}{A_{H} \rho C_{p} v_{H}}
\end{aligned}
$$

and substitute above:

$$
\frac{\partial T_{i}}{\partial x}+\alpha\left(T_{i}-T_{i-1}\right)-\alpha\left(T_{i+1}-T_{i}\right)+\beta\left(T_{i}-T_{H}\right)=0
$$

for $i=1 \ldots N$, and

$$
\frac{\partial T_{H}}{\partial x}-\sum_{i=1}^{N} \gamma\left(T_{i}-T_{H}\right)=0
$$

Average temperature equations bundle-hole coupling

We now focus first on the average temperature of the cable bundle, given by:

$$
\bar{T}=\frac{1}{N} \sum_{i=1}^{N} T_{i}
$$

An equation for the average temperature can be obtained adding all equations for the single bundle channels, i.e. sum all the Eqs. (26)

$$
\frac{\partial \bar{T}}{\partial x}+\beta \bar{T}-\beta T_{H}=0
$$

that contains only the average bundle temperature and the hole temperature. We note in Eq. (27) that we can also write:

$$
\frac{\partial T_{H}}{\partial x}-N \gamma \bar{T}+N \gamma T_{H}=0
$$


Equations (29) and (30) form a closed system of ODEs, with initial conditions known from Eqs. (19) and (20):

$$
\begin{aligned}
& \bar{T}(0)=T_{0}+\Delta T / N \\
& T_{H}(0)=T_{0}
\end{aligned}
$$

We can write the system of equations for the average and hole temperature as follows:

$$
\frac{\partial}{\partial x}\left(\begin{array}{c}
\bar{T} \\
T_{H}
\end{array}\right)+\left(\begin{array}{cc}
\beta & -\beta \\
-N \gamma & N \gamma
\end{array}\right)\left(\begin{array}{c}
\bar{T} \\
T_{H}
\end{array}\right)=0
$$

which can be solved using the general formalism discussed below.

Local temperature differences bundle-bundle coupling

To obtain the single temperatures in the bundle channels, we define the temperature difference:

$$
\Delta T_{i}=T_{i}-\bar{T}
$$

and we subtract the equation for the average temperature, Eq. (29), from the equation for the individual bundle channels, Eq. (26), obtaining:

$$
\frac{\partial \Delta T_{i}}{\partial x}+-\alpha \Delta T_{i-1}+(2 \alpha+\beta) \Delta T_{i}-\alpha \Delta T_{i+1}=0
$$

that has initial conditions:

$$
\Delta T_{i}(0)=\left\{\begin{array}{lc}
\frac{N-1}{N} \Delta T & \text { for } i=1 \\
-\frac{1}{N} \Delta T & \text { for } 2 \leq i \leq N
\end{array}\right.
$$

The system of Eqs. (34) can be written as follows:

$$
\frac{\partial}{\partial x}\left(\begin{array}{c}
\Delta T_{1} \\
\Delta T_{2} \\
\Delta T_{3} \\
\vdots \\
\Delta T_{N}
\end{array}\right)+\left(\begin{array}{ccccc}
2 \alpha+\beta & -\alpha & 0 & \cdots & -\alpha \\
-\alpha & 2 \alpha+\beta & -\alpha & \cdots & 0 \\
0 & -\alpha & 2 \alpha+\beta & \cdots & 0 \\
\vdots & \vdots & \vdots & \ddots & 0 \\
-\alpha & 0 & 0 & \cdots & 2 \alpha+\beta
\end{array}\right)\left(\begin{array}{c}
\Delta T_{1} \\
\Delta T_{2} \\
\Delta T_{3} \\
\vdots \\
\Delta T_{N}
\end{array}\right)=0
$$

that we solve with the formalism discussed below. We remark here that the matrix of coefficients in Eq. (36) is symmetric and circulant.

General solution method

To find a solution we represent the system of Eqs. (32) and (36) as follows: 


$$
\begin{aligned}
\frac{\partial y}{\partial x}+A y & =0 \\
y(0) & =y_{0}
\end{aligned}
$$

where $y$ and $A$ are a vector and a matrix respectively, of dimension M. We indicate with $\Lambda$ the matrix of eigenvalues of $A$, and with $Y$ the matrix of eigenvectors, such that:

$$
A Y=\Lambda Y
$$

If we indicate with $\lambda_{k}$ a diagonal element of the matrix $\Lambda$ and with $y_{k}$ a column of the matrix $Y$, then the general solution to the system of homogeneous ODE is:

$$
y=\sum_{k=1}^{M} c_{k} y_{k} e^{-\lambda_{k} x}
$$

where the $c_{k}$ are the integration constant that need to be determined based on the initial condition Eqs. (38-38), and can be interpreted as the amplitudes of each mode. Below we proceed to the solution of the average and hole temperature, and of the temperature differences.

Solution for the average bundle and hole temperature

The vector $y$ is given by:

$$
y=\left(\begin{array}{c}
\bar{T} \\
T_{H}
\end{array}\right)
$$

the matrix $A$ is given by:

$$
A=\left(\begin{array}{cc}
\beta & -\beta \\
-N \gamma & N \gamma
\end{array}\right)
$$

and the initial conditions $y_{0}$ are:

$$
y_{0}=\left(\begin{array}{c}
T_{0}+\Delta T / N \\
T_{0}
\end{array}\right)
$$

The eigenvalues and eigenvectors of the matrix $A$ are:

$$
\Lambda=\left(\begin{array}{cc}
0 & 0 \\
0 & N \gamma+\beta
\end{array}\right)
$$




$$
Y=\left(\begin{array}{cc}
1 & 1 \\
1 & -N \gamma / \beta
\end{array}\right)
$$

Following Eq. (40), the solution can be hence written:

$$
y=c_{1}+c_{2}\left(\begin{array}{c}
1 \\
-\frac{N \gamma}{\beta}
\end{array}\right) e^{-(\beta+N \gamma) x}
$$

To satisfy Eq. (43) to the initial conditions, the constants must have values:

$$
\begin{aligned}
c_{1} & =T_{0}+\frac{\frac{N \gamma}{\beta}}{1+\frac{N \gamma}{\beta}} \frac{\Delta T}{N} \\
c_{2} & =\frac{1}{1+\frac{N \gamma}{\beta}} \frac{\Delta T}{N}
\end{aligned}
$$

The general result contains two modes. The first is a constant, and representes the asymptotic temperature of the system (i.e. the exit temperature after an infinite length). The second mode is a non-symmetric temperature difference and has eigenvalue:

$$
\lambda_{2}=N \gamma+\beta
$$

This is the inverse of the characteristic length for the average temperature distribution, which is not affected by the temperature differences among the single subcable channels in the bundle.

Solution for the local temperature differences

In the case of the bundle channels, the vector $y$ is given by:

$$
y=\left(\begin{array}{c}
\Delta T_{1} \\
\Delta T_{2} \\
\Delta T_{3} \\
\vdots \\
\Delta T_{N}
\end{array}\right)
$$

the matrix $A$ is given by:

$$
A=\left(\begin{array}{ccccc}
2 \alpha+\beta & -\alpha & 0 & \cdots & -\alpha \\
-\alpha & 2 \alpha+\beta & -\alpha & \cdots & 0 \\
0 & -\alpha & 2 \alpha+\beta & \cdots & 0 \\
\vdots & \vdots & \vdots & \ddots & 0 \\
-\alpha & 0 & 0 & \cdots & 2 \alpha+\beta
\end{array}\right)
$$


and the initial conditions $y_{0}$ are:

$$
y_{0}=\left(\begin{array}{c}
\frac{N-1}{N} \Delta T \\
-\frac{\Delta T}{N} \\
-\frac{\Delta T}{N} \\
\vdots \\
-\frac{\Delta T}{N}
\end{array}\right)
$$

The matrix $A$ is sparse, symmetric and circulant. The eigenvectors are the discrete Fourier transform of same dimension, while the eigenvalues are the associated frequencies. For the specific form of matrix $A$, we distinguish two cases, i.e. an even and an odd value of $N$, as follows:

\section{N even}

$$
\begin{gathered}
p=\frac{N}{2} \\
\lambda_{k}=2 \alpha\left(1-\cos \frac{\pi(k-p)}{p}\right)+\beta
\end{gathered}
$$

with $k=1 \ldots N$

$$
\begin{aligned}
y_{N / 2-q}^{T} & =\sqrt{\frac{2}{N}}\left[\begin{array}{lllll}
\sin 2 \pi q \frac{N-1}{N} & \sin 2 \pi q \frac{N-2}{N} & \cdots & \sin 2 \pi q \frac{1}{N} & 0
\end{array}\right] \\
y_{N / 2}^{T} & =\sqrt{\frac{2}{N}}\left[\begin{array}{lllll}
1 & 1 & \cdots & 1 & 1
\end{array}\right] \\
y_{N / 2+q}^{T} & =\sqrt{\frac{2}{N}}\left[\begin{array}{lllll}
\cos 2 \pi q \frac{N-1}{N} & \cos 2 \pi q \frac{N-2}{N} & \cdots & \cos 2 \pi q \frac{1}{N} & 1
\end{array}\right] \\
y_{N}^{T} & =\sqrt{\frac{2}{N}}\left[\begin{array}{lllll}
1 & -1 & \cdots & 1 & -1
\end{array}\right]
\end{aligned}
$$

with $q=1 \ldots p-1$

$\underline{N \text { odd }}$

$$
\begin{gathered}
p=\frac{N-1}{2} \\
\lambda_{k}=2 \alpha\left(1-\cos \frac{2 \pi(k-p)}{2 p+1}\right)+\beta
\end{gathered}
$$

with $k=1 \ldots N$ 


$$
\begin{aligned}
& y_{(N+1) / 2-q}^{T}=\sqrt{\frac{2}{N}}\left[\begin{array}{lllll}
\sin 2 \pi q \frac{N-1}{N} & \sin 2 \pi q \frac{N-2}{N} & \cdots & \sin 2 \pi q \frac{1}{N} & 0
\end{array}\right] \\
& y_{(N+1) / 2}^{T}=\sqrt{\frac{2}{N}}\left[\begin{array}{lllll}
1 & 1 & \cdots & 1 & 1
\end{array}\right] \\
& y_{(N+1) / 2+q}^{T}=\sqrt{\frac{2}{N}}\left[\begin{array}{lllll}
\cos 2 \pi q \frac{N-1}{N} & \cos 2 \pi q \frac{N-2}{N} & \cdots & \cos 2 \pi q \frac{1}{N} & 1
\end{array}\right]
\end{aligned}
$$

with $q=1 \ldots p$

At this point the general solution of the system of ODEs is a matter of simple, but tedious algebra.

We simplify further the discussion assuming that $\mathrm{N}$ is even (in our case $N=6$ channels under the external thermometers), and we hence only deal with the eigenvalues and eigenvectors given by Eqs. (53-58). At this point it is interesting to examine the eigenvalues and the eigenvectors, each of them representing a characteristic length and a mode in the temperature difference evolution. The eigenvalues form an ordered series spanning the range $(\alpha+\beta \ldots 4 \alpha+\beta)$. In the case $N=6$ we obtain the following values:

$$
\begin{aligned}
& \lambda_{1}=3 \alpha+\beta \\
& \lambda_{2}=2 \alpha+\beta \\
& \lambda_{3}=\alpha+\beta \\
& \lambda_{4}=2 \alpha+\beta \\
& \lambda_{5}=3 \alpha+\beta \\
& \lambda_{6}=4 \alpha+\beta
\end{aligned}
$$

where we remark that apart for the smallest and the largest, all other values are paired, and correspond to the eigenvectors obtained from odd or even functions. We have plotted in Fig. 29 the corresponding sequence of eigenvectors. The mode with the lowest eigenvalue (longest decay length) is uniform, while the other eigenvectors are pairs functions with 1 or 2 periods with increasing eigenvalues. The last eigenvector, with the highest eigenvalue, also has the highest periodicity.

We see here the evident analogy between the eigenvectors and the sin and cos functions that form the basis of a Fourier decomposition of the temperature differences over the number $N$ of parallel channels. Indeed, it can be shown that the eigenvectors with a given eigenvalue have non-zero projection only on the Fourier components (sin and cos functions) that have the same period. This is very relevant to the analysis of the results measured, where we have performed Fourier analysis to derive the first order component of the temperature differences. According to the discussion above, this mode corresponds to the two coincident eigenvalues:

$$
\lambda_{2}=\lambda_{4}=2 \alpha+\beta
$$


whose value is the inverse of the characteristic length of the evolution of the first Fourier component of the temperature differences.

For what regards the general solution of the ODE, the amplitudes of the eigenmodes can be obtained analytically, as done for the case of average temperatures. In practice the treatment is rather lengthy, and we prefer to limit ourselves to saying that the values of the integration constants are obtained solving the system:

$$
Y c=y_{0}
$$

where $Y$ is the matrix of eigenvectors obtained composing the column vectors specified in Eqs. (62-63), $y_{0}$ is the column vector of the initial values, as given in Eq. (52), and $c$ is the column vectors of the integration constants $c_{k}$. The above result is general, and applies to any number of channels $N$. We also remark that the solution for the temperature differences cannot contain a uniform temperature increase with respect to the average temperature. This means that the eigenmode of order $N / 2$, i.e. the one with the lowest eigenvalue, must necessarily have zero amplitude in the general solution, and a zero integration constant $c_{N / 2}$.

We show below an example of analytical solution obtained using the above method.

\section{Example of analytical solution}

The above equations have been solved for the parameters reported in Tab. 4, which are relevant to the study performed here. We compare there the analytical solution to a numerical integration used as benchmark. As expected the two agree perfectly.

The series of eigenvalues is reported in the inset for reference.

\section{Summary}

In summary, we have shown above that the average temperature difference between bundle and hole varies along the length with an eigenvalue given by Eq. (49). In terms of the original varibles, this corresponds to an inverse characteristic length:

$$
\bar{\lambda}=k_{B H}=p_{B H} h_{B H}\left(\frac{1}{A_{B} \rho C_{p} v_{B}}+\frac{N}{A_{H} \rho C_{p} v_{H}}\right)
$$

The temperature differences in the bundle can be expressed in terms of a sum of periodic functions over the number of channels. Each frequency corresponds to a different eigenvalue. The eigenvalue for the first period is given by Eq. (70), and, in terms of the original variables, corresponds to an inverse characteristic length:

$$
\lambda_{\Delta}=k_{B B}=2 \frac{p_{B B} h_{B B}}{A_{B} \rho C_{p} v_{B}}+\frac{p_{B H} h_{B H}}{A_{B} \rho C_{p} v_{B}}
$$


Table 4: Parameters used for the analytical solution.

\begin{tabular}{lccc}
\hline Description & Symbol & Value & Unit \\
\hline Total helium in bundle & $A_{B t o t}$ & 300.0 & $\mathrm{~mm}^{2}$ \\
Helium in central channel (hole) & $A_{H}$ & 100.0 & $\mathrm{~mm}^{2}$ \\
Wetted perimeter bundle/bundle & $p_{B B}$ & 5.0 & $\mathrm{~mm}$ \\
Total wetted perimeter bundle/hole & $p_{B H t o t}$ & 10.0 & $\mathrm{~mm}$ \\
Radial heat transfer coefficient & $h_{B H}$ & 400 & $\mathrm{~W} / \mathrm{m}^{2} \mathrm{~K}$ \\
Azimuthal heat transfer coefficient & $h_{B B}$ & 200 & $\mathrm{~W} / \mathrm{m}^{2} \mathrm{~K}$ \\
Helium velocity in bundle & $v_{B}$ & 0.1 & $\mathrm{~m} / \mathrm{s}$ \\
Helium velocity in hole & $v_{H}$ & 0.5 & $\mathrm{~m} / \mathrm{s}$ \\
Helium density & $\rho$ & 100 & $\mathrm{~kg} / \mathrm{m}^{3}$ \\
Helium specific heat at const. pressure & $C_{p}$ & 3000 & $\mathrm{~J} / \mathrm{kg} / \mathrm{K}$ \\
\hline
\end{tabular}



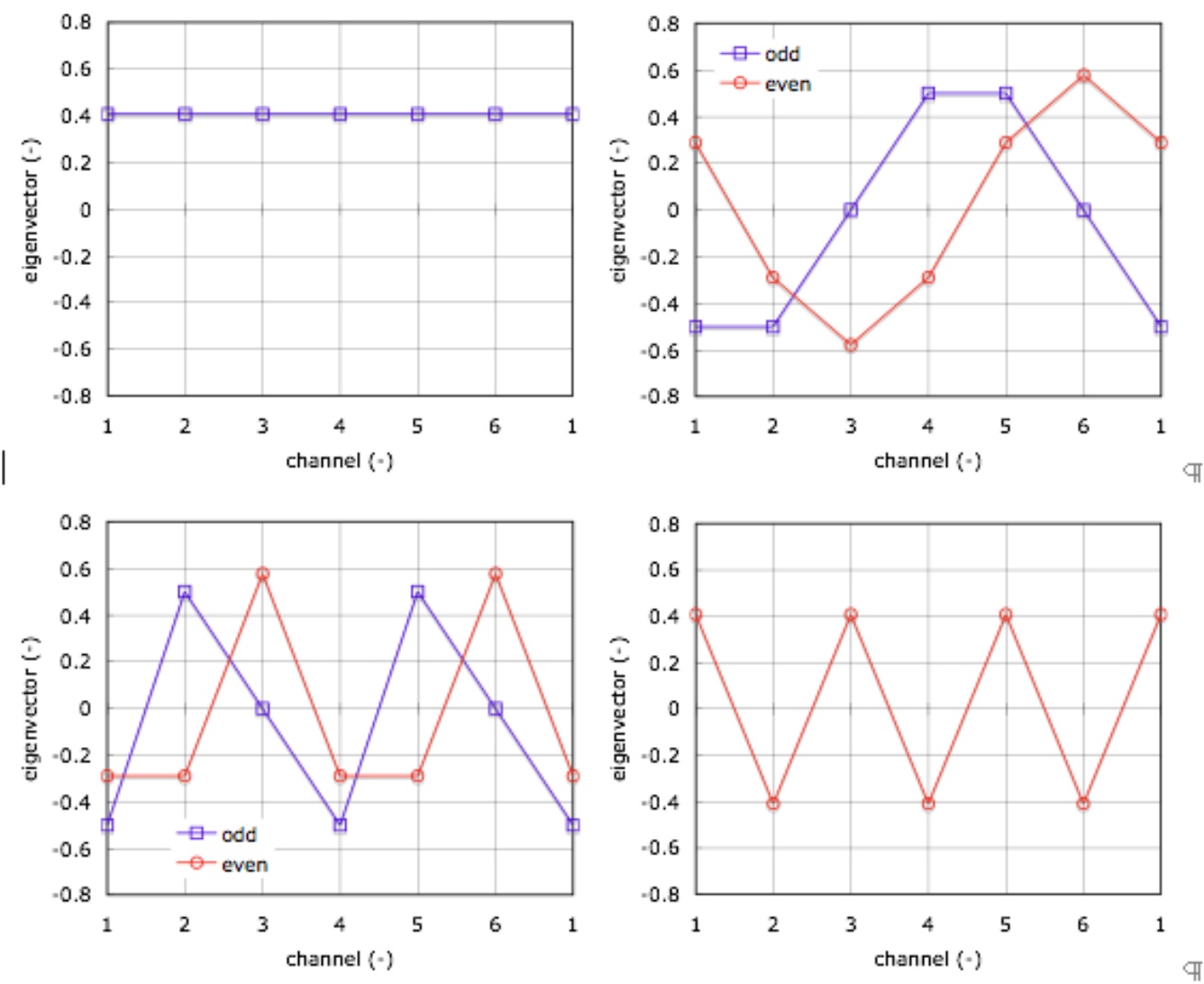

Figure 29: Series of eigenvectors obtained analytically for the case $\mathrm{N}=6$, ordered by eigenvalue (from top-left to bottom-right). The two eigenvalues in the center are multiple, and have two associated eigenvectors each. 


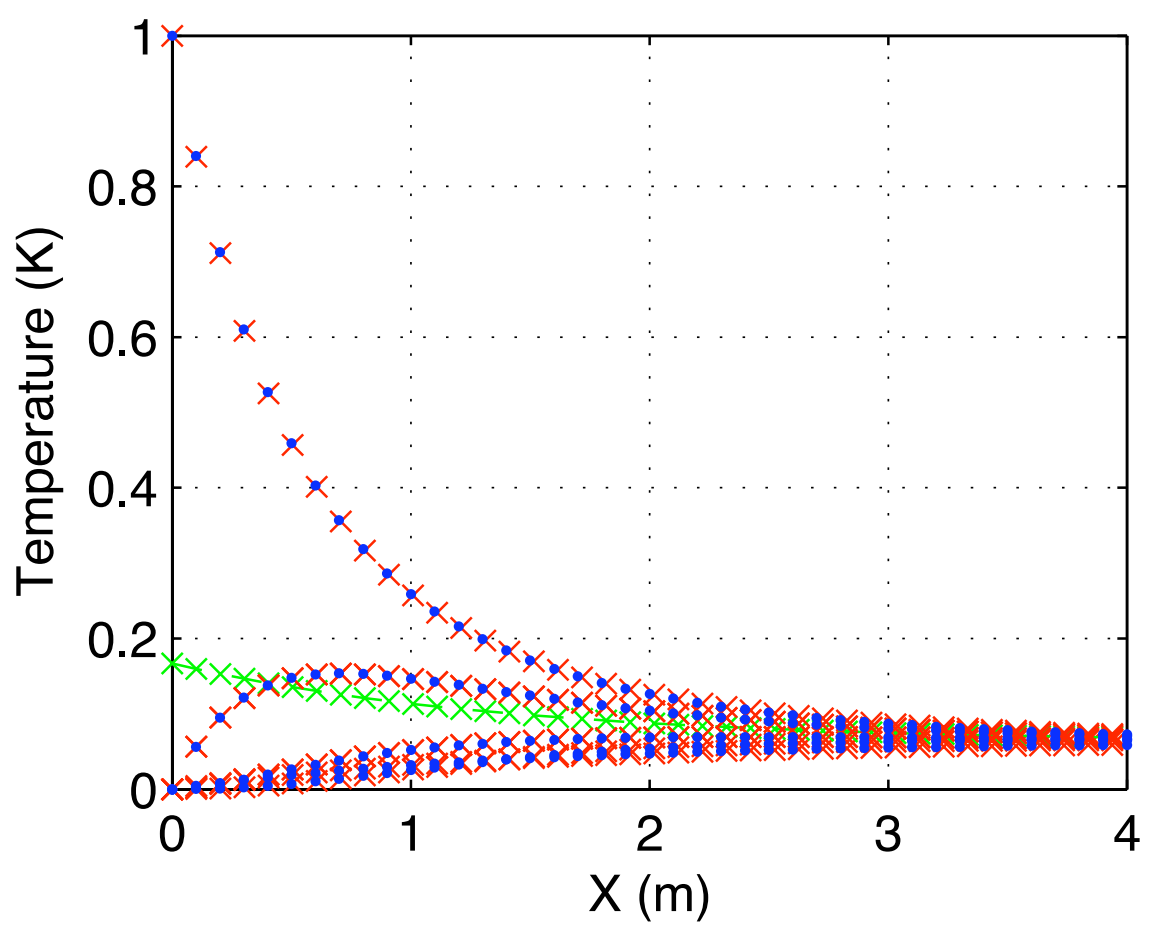

Figure 30: Comparison of analytical solution (symbol x-red) and numerical solution (symbol dot-blue). The curve $x$-green is the average temperature of the bundle. 


\section{Appendix B}

\section{Fourier Transform of the azimuthal temperature distribution}

The Fast Fourier Transform of the azimuthal temperature distribution $\Delta T_{i j}$ is:

$$
f_{i}=f_{F F T}\left(\Delta T_{i j}\right)
$$

There are $\mathrm{N}=6$ sensors on the $i$ th ring, and therefore a total of 6 Fourier coefficients:

$$
\begin{aligned}
A 1_{i} & =(1 / N) \operatorname{Re}\left(f_{i}(1)\right) \\
A 2_{i} & =(2 / N) \operatorname{Re}\left(f_{i}(2)\right) \\
A 3_{i} & =(2 / N) \operatorname{Re}\left(f_{i}(3)\right) \\
A 4_{i} & =(1 / N) \operatorname{Re}\left(f_{i}(4)\right) \\
B 2_{i} & =(2 / N) \operatorname{Im}\left(f_{i}(2)\right) \\
B 3_{i} & =(2 / N) \operatorname{Im}\left(f_{i}(3)\right)
\end{aligned}
$$

The inverse function and the reconstructed function are, respectively:

$$
\begin{aligned}
\text { finv }_{i} & =A 1_{i}+A 2_{i} \cos \phi+A 3_{i} \cos 2 \phi+A 4_{i} \cos 3 \phi-B 2_{i} \sin \phi-B 3_{i} \sin 2 \phi \\
\text { frec }_{i} & =A 1_{i}+A 2_{i} \cos \phi-B 2_{i} \sin \phi
\end{aligned}
$$

The modules of the Fourier coefficients are:

$$
\begin{aligned}
a b s A 1_{i} & =A 1_{i} \\
a b s A 2_{i} & =(2 / N)\left|f_{i}(2)\right| \\
a b s A 3_{i} & =(2 / N)\left|f_{i}(3)\right| \\
a b s A 4_{i} & =(1 / N)\left|f_{i}(4)\right|
\end{aligned}
$$

Details are given in [12]. 


\section{Appendix C}

\section{Porous media analogy}

To interpret the experimental data collected, we postulate an analogy between the flow in the cable bundle of a CICC and the theory of mass, momentum and energy convection in porous media [13]. The cabled strands form the packed solid phase that obstructs the free flow in the channel. The pores are the interstices between strands, and the coolant flows in the tortuous path connecting the interstices. The porosity $\varphi$, i.e. the ratio between the fluid volume to the total volume, is identical to the void fraction of the cable. Finally, the characteristic size of the porous media is the strand diameter.

A similar approach was followed by Long [19], who went into extensive details to obtain friction factor and heat transfer correlations. In the following sections we limit to a review of the basic assumptions and results that can be derived from a theory of convection in porous media, restricting the discussion to the results that are relevant to forced convection in CICC's.

Pressure drop

The momentum balance of a fluid in a porous medium can be written in the following form, using the Dupuit-Forcheimer modification to the Darcy's equation for the gradient of pressure [13]:

$$
\nabla p=-\frac{\mu}{K} \mathbf{v}_{\varphi}-c_{F} \frac{\rho_{F}}{K^{1 / 2}}\left|\mathbf{v}_{\varphi}\right| \mathbf{v}_{\varphi}
$$

where the first term is the low-speed linear drag relation established by Darcy, and the second term, often referred to as Forcheimer term, was establishd in the form presented above by Ward [18]. Above we indicate with $\mathbf{v}_{\varphi}$ the vector of seepage velocity, which is related to the average fluid velocity $\mathbf{v}$ by: ${ }^{3}$

$$
\mathbf{v}_{\varphi}=\varphi \mathbf{v}
$$

$\rho_{F}$ is the fluid density, $c_{F}$ is a dimensionless form-drag constant and $K$ is the permeability (assumed to be isotropic). In the case of particle or fiber beds, the permeability $K$ is given

\footnotetext{
${ }^{3}$ In the discussion we need to use three concepts of fluid velocity, corresponding to three different scales of volumes. At the first level, at a microscopic scale much larger than the molecular length, we can define the intrinsic velocity of the fluid $\mathbf{V}$, that can be different from point to point within a pore. This is the velocity that describes the 3-D flow field in the pores, and within a pore. The equations of flow are generally written for an average fluid velocity, $\mathbf{v}$, that is obtained averaging the intrinsic velocity $\mathbf{V}$ over a representative elementary volume of fluid $V_{F}$ that excludes the solid phase, and whose size is large enough to produce a values independent on the volume itself. In practice $V_{F}$ must be larger than the pore size, but smaller than the length scale of the macroscopic flow domain. The balances of mass, momentum and energy require finally dealing with averages over a volume $V$ of size comparable to $V_{F}$, but including both fluid and solid phases. The result of this averaging process produces fluid quantity, including the seepage velocity $\mathbf{v}_{\varphi}$, that are weighted by the porosity $\varphi$.
} 
by the following expression, attributed to Carman-Kozeny:

$$
K=\frac{D_{p}^{2} \varphi^{3}}{180(1-\varphi)^{2}}
$$

where $D_{p}$ is the characteristic size of the solid phase, e.g. the particle diameter in a bed of packed spheres. If we further consider only a single component of the flow (e.g. the $x$ direction), the momentum balance can be written as:

$$
\frac{\partial p}{\partial x}=-\frac{\mu}{K} v_{\varphi}-c_{F} \frac{\rho_{F}}{K^{1 / 2}}\left|v_{\varphi}\right| v_{\varphi}
$$

where $\mu$ is the fluid viscosity. To derive results applicable for the pressure drop in CICC's, we wish to collect the terms in the above expression to produce the relation commonly used for the pressure drop, i.e. involving a friction factor $f$ :

$$
\frac{\partial p}{\partial x}=-2 \frac{f}{D_{h}} \rho_{F}|v| v
$$

where $D_{h}$ is the hydraulic diameter. Comparing the two expressions above, we obtain:

$$
f=\frac{c_{F} D_{h}}{2 \varphi^{2} K^{1 / 2}}+\frac{D_{h}}{2 \varphi K} \frac{\mu}{\rho_{F}|v|}
$$

which can be also written in the following form:

$$
f=a(\varphi)+\frac{b(\varphi)}{\operatorname{Re}}
$$

We remark that the above expression resembles closely the friction factor fit produced by Katheder [7] using as a starting point correlations for pressure drop in beds of glass spheres.

Thermal dispersion

A peculiarity of forced convection in porous media is that the heat transfer within the fluid and from the fluid to the solid is strongly affected by mixing of the fluid. This is a direct consequence of the tortuous flow path in the porous medium. Mixing is caused by the particular structure of the flow paths, with blocked or confined channels, by recirculation and eddy effects at flow restrictions. Furthermore, macroscopic mixing takes place at the interstitial (pore) scale.

To elucidate this process, we can picture fluid elements starting at a distance from each other, and flowing in different channels. Because of the tortuosity of the channels, the fluid elements will not remain at the same distance apart along the flow path. The net effect is a two-ways macroscopic transport of mass that promotes mixing of fluid over distances much larger than the molecular length. In the presence of temperature gradients, this effect also 
results in heat transfer that, depending on the flow conditions, can be largely in excess of molecular diffusion. In the literature this phenomenon is referred to as thermal dispersion, and is characterised by an effective thermal conductivity.

An accurate description of thermal dispersion is a complex mathematical and physical matter. We consider here the case of isotropic medium and 1-D flow, and we limit ourselves to simple approximations for the effective longitudinal and transverse thermal conductivity that are obtained for beds of uniform spheres.

A suitable expression for the effective longitudinal conductivity, i.e. in the direction of the flow, is [13]:

$$
k_{/ /}=(1-\varphi) k_{S}+\varphi \frac{2 B}{\pi} P e_{/ /} k_{F}
$$

Above, $k_{F}$ and $k_{S}$ is the thermal conductivity in fluid and solid, respectively, $B$ is a constant (empirically $B=1.75$ ) and the longitudinal Peclet number is defined as:

$$
P e_{/ /}=\frac{v_{\varphi} D_{p}}{\alpha_{F}(1-\varphi)}
$$

where $\alpha_{F}$ is the thermal diffusivity of the fluid:

$$
\alpha_{F}=\frac{k_{F}}{\rho_{F} C_{p F}}
$$

In the direction transverse to the flow, which is most relevant for the heat transfer experiments discussed here, the effective thermal conductivity has been derived theoretically and experimentally, leading to several expressions depending on the approach taken. The simplest expression was obtained correlating experimental data:

$$
k_{\perp}=k_{F} C_{T} P e_{\perp}
$$

where $C_{T}$ is a constant (empirically, $C_{T}=0.09 \ldots 0.10$ ).

At high Reynolds number, Hsu et al. have provided a theoretical support for the above expression, obtained by volume averaging of the flow and temperature deviations from average [14]:

$$
k_{\perp}=k_{F} D_{T} \frac{1-\varphi}{\varphi} P e_{\perp}
$$

where the value of the constant $D_{T}$ is also determined experimentaly (a good value is $D_{T}=$ 0.04 ), and the transverse Peclet number is computed as: 


$$
P e_{\perp}=\frac{v_{\varphi} D_{p}}{\alpha_{F}}
$$

An alternative approach was followed by Ming et al. [16], using a fractal model to describe the tortuosity of the flow in a porous medium. Defining the ratio of the straight distance $L_{s}$ travelled by a particle (the distance of a coherent macroscoptic motion) to the size of the pore $\lambda_{\min }$ (the smallest scale of the movement), and the fractal dimension of the tortuous path $\delta$ (where $1<\delta<2$ ) they found that:

$$
\delta=1+\frac{\ln (1+\sqrt{1-\varphi})}{\ln \left(L_{s} / \lambda_{\min }\right)}
$$

and that the effective transverse conductivity is given by:

$$
k_{\perp}=k_{F} E_{T} \frac{1}{\varphi}\left[\delta\left(\frac{L_{s}}{\lambda_{\min }}\right)^{\delta-1}-1\right] P e_{\perp}
$$

where the constant $E_{T}$ is based on experimental data, and a value $E_{T}=0.03$ yields consistent results to the work reported above.

\section{Heat transfer coefficients}

We can pursue the analogy to porous media flow to give estimates of the heat transfer coefficient. We distinguish here in particular two different heat transfer coefficients: the coefficient $h_{\text {ext }}$ characterizing the heat transfer from the boundary of the flow to the flow bulk, and the coefficient $h_{\text {int }}$ characterizing the heat transfer from the solid phase to the fluid phase at the pore level.

In general, as discussed in [13], both heat transfer coefficients can be obtained as from classical correlations, modified as follows to take into account the increased thermal conductivity as resulting from thermal dispersion:

$$
\frac{h^{\text {porous }}}{h^{\text {free }}}=\frac{k_{F}+k_{\perp}}{k_{F}}
$$

where $h^{\text {porous }}$ indicates the heat transfer coefficient in the porous flow, and $h^{\text {free }}$ is the heat transfer coefficient as would be obtained in the same configuration, but considering free flow under the same conditions.

In practice, for the external heat transfer coefficient, it is possible to use correlations of the same type as used in an equivalent smooth tube, corrected by the ratio above. A suitable correlation for a pipe of diameter $D$ is [17]:

$$
\frac{h_{\text {ext }} D}{k_{F}+k_{\perp}}= \begin{cases}8 & \text { for laminar flow } \\ 0.023 \operatorname{Re}_{D}^{0.8} \operatorname{Pr}^{0.4} & \text { for turbulent flow }\end{cases}
$$


where the Reynolds number $R e_{D}$ is naturally referred to the pipe diameter.

The internal heat transfer, in addition to the above correction, also obeys specific correlations that exhibit higher Nusselt number and stronger dependence on Reynods number than the typical correlations used for flow in smooth tubes. Examples can be found in [15] for different geometries and porosity. In particular, the correlation for a bed of packed spheres of diameter $D_{p}$ is:

$$
\frac{h_{e x t} D_{p}}{k_{F}+k_{\perp}}=0.093 \operatorname{Re}_{D_{p}}^{1.04} \operatorname{Pr}^{1 / 3}
$$

where we remark that in this case the Reynolds number $R e_{D p}$ is referred to the sphere diameter.

\section{Channeling and porosity variations}

So far we have considered a uniform and unbounded medium. This is not appropriate in the case of the superconducting cable considered. While the medium is indeed unbounded in longitudinal direction, and can be considered unbounded along the perimeter (it is periodic), it has two discontinuities in radial direction: the cooling channel at the inner radius and the jacket at the outer radius. As discussed in [13] and [14], this results in a local change of porosity which causes channeling effects (increased local massflow at the two boundaries) and a modification of the mixing properties discussed above. This is important as it shows the limit of the results discussed so far.

The effective porosity at a distance $r$ from the boundary is described for convenience by an exponential function of the form:

$$
\varphi=\varphi_{\infty}\left[1+C_{1} e^{-\frac{N_{1} r}{D_{p}}}\right]
$$

where $C_{1}$ and $N_{1}$ are suitable fitting constants, and $\varphi_{\infty}$ is the porosity in the homogeneous portion of the porous medium, away from the boundary. With a value of $C_{1}=1.4$ and $N_{1}=5$, as appropriate for packed beds, this means that the porosity at the wall is more then double the nominal value, and the dimension affected is of the order of five times the characteristic size of the solid phase. The flow in this region is much less constricted (channeling), which explains the steep temperature gradients observed near the heated or cooled wall of a packed column.

Following Hsu et al., [14], a modification of the local value of the porosity also leads to a change in the effective transverse conductivity. A suitable expression for the local value of the transverse conductivity is:

$$
k_{\perp}=k_{F} C_{2} P e_{\perp} l\left(\frac{v_{\varphi}^{l o c a l}}{v_{\varphi}}\right)
$$

where we have introduced a wall function: 


$$
l=1-e^{-\frac{N_{2} r}{D_{p}}}
$$

and the ratio $\frac{\nu_{\varphi}^{\text {local }}}{\nu \varphi}$ of local to average seepage velocity. The fitting constants in this case have values $C_{2}=0.12$ and $N_{2}=1$, which implies that the thermal conductivity is modified only over a length of the same scale as the characteristic dimension of the solid phase. 


\section{List of Symbols}

All symbols used in the text and a selection of symbols used in Appendix A, B and C are described here. The remaining symbols are described in the Appendices. The subscript $B$ is used for the bundle, the subscript $\mathrm{H}$ for the hole (central spiral).

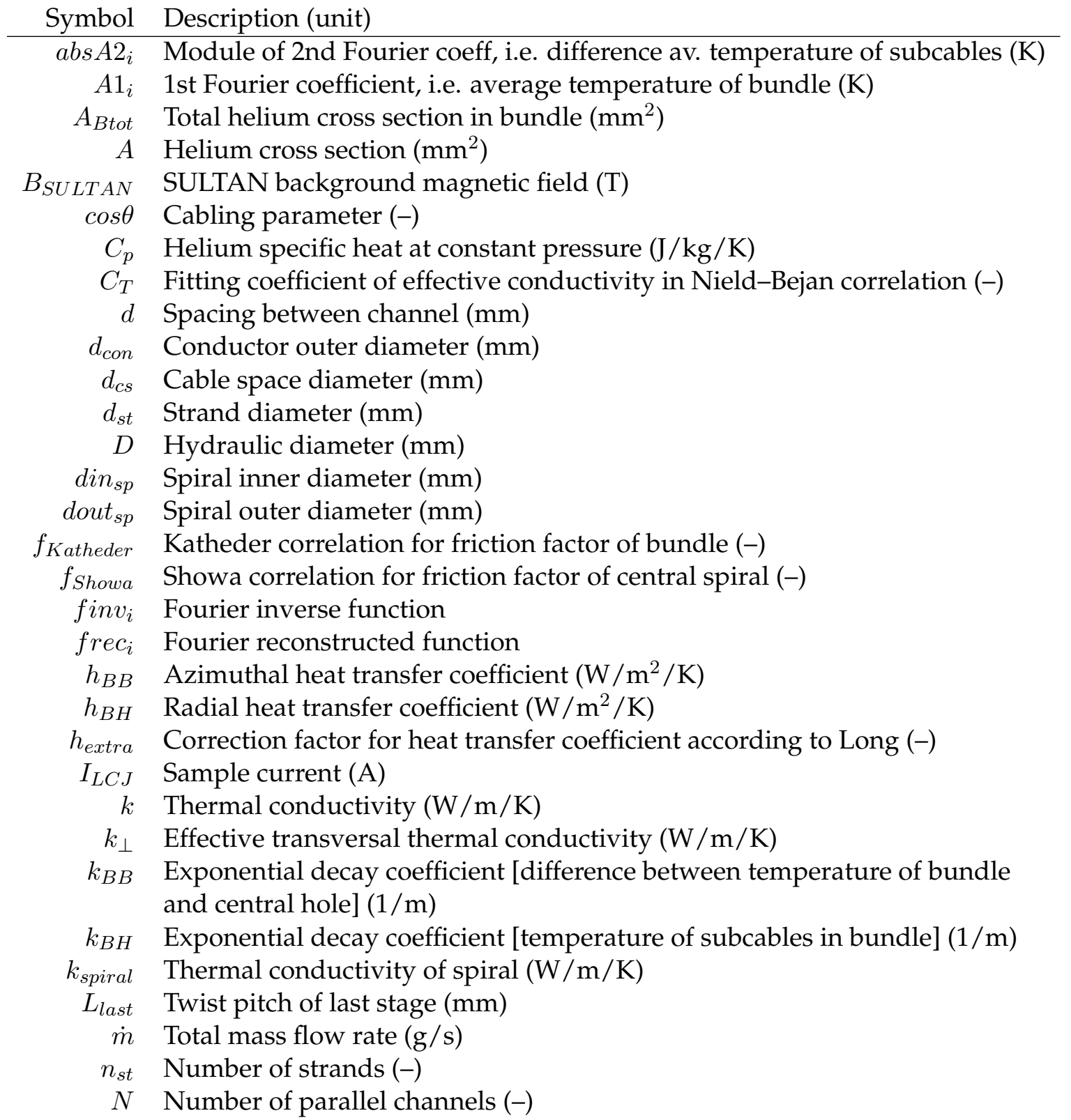




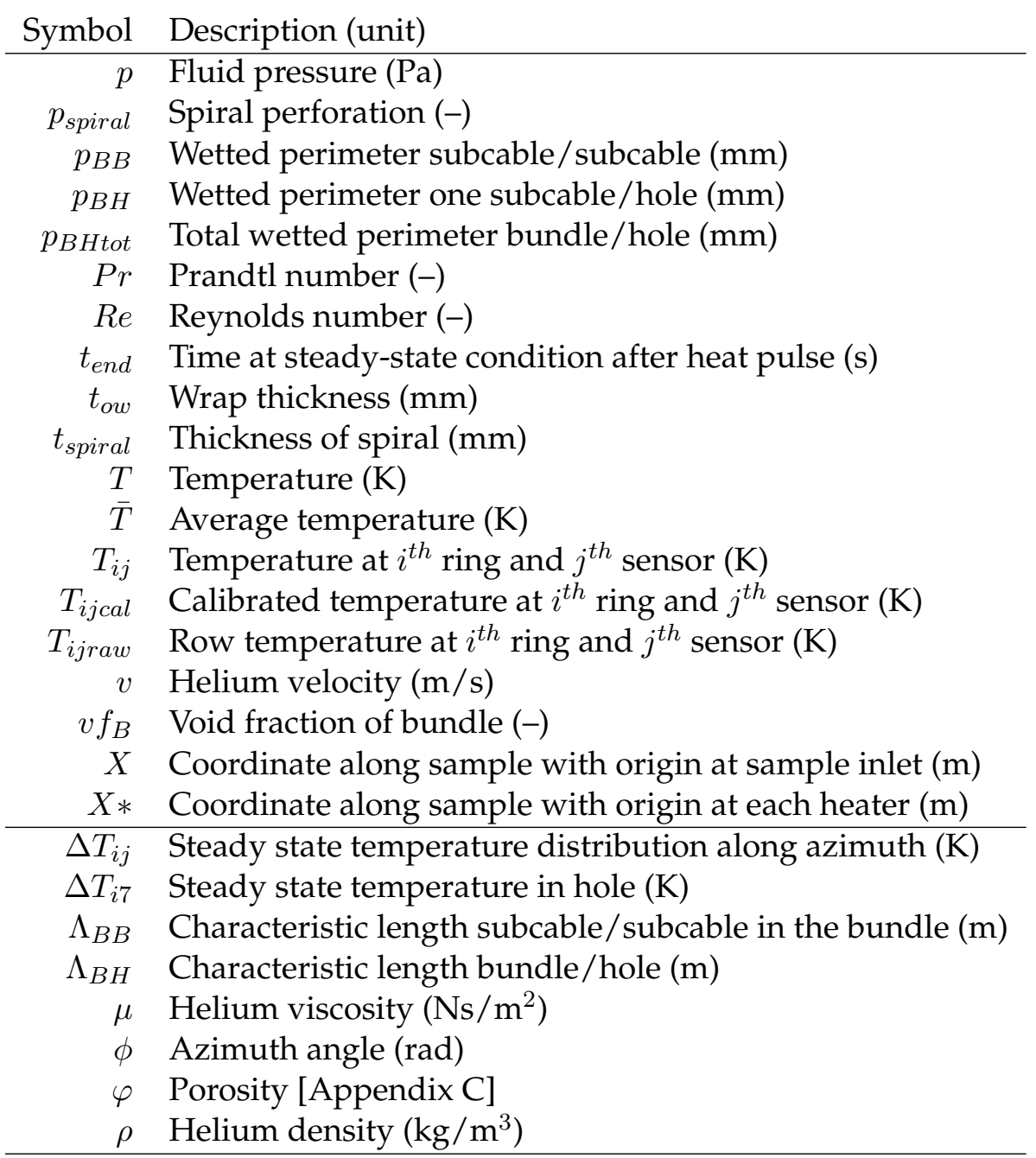




\section{List of Tables}

1 Geometric parameters used for the thermal hydraulic analysis of the LCJ sample. The data in the first part of the Table are from the specifications ([1], [2]), those in the second part are deduced. . . . . . . . . . . . . . . .

2 Summary of thermal hydraulic runs (TH) and calibration runs of the LCJ sample. All runs are performed at $B_{S U L T A N}=0$ and $I_{L C J}=0$. The helium initial conditions at the sample inlet are: $4.5 \mathrm{~K}$ and 1.0MPa. The runs LCJH270907, LCJH280911 LCJH290909 were used to calibrate all TH measurements of the same day. The results of heater $\mathrm{H} 3$ at $4 \mathrm{~g} / \mathrm{s}$ and $6 \mathrm{~g} / \mathrm{s}$ are not consistent with the other results of the measurement set and therefore have been rejected in this report. . . . . . . . . . . . . . . . . .

3 Summary of results, i.e. exponent of Eq. (4) and Eq. (5) and transverse heat transfer coefficients in Eq. (8) and Eq. (10), using the nominal friction factors, i.e. $f_{B}=0.7 f_{\text {Katheder }}$ and $f_{H}=f_{\text {Showa }} \ldots \ldots \ldots \ldots \ldots$

4 Parameters used for the analytical solution. . . . . . . . . . . . . . . . .

\section{List of Figures}

1 Cross section of the Low Cost Joint conductor. . . . . . . . . . . . . . . .

2 Schematic build up of the Low Cost Joint conductor, with details of the central spiral. . . . . . . . . . . . . . . . . . . . . .

3 Schematic experimental set-up of the LCJ sample in SULTAN. Helium enters the sample from the bottom, is heated up by 5 resistive heaters $(\mathrm{H} 0, \mathrm{H} 3, \mathrm{H} 7$, $\mathrm{H} 8, \mathrm{H} 9)$ and the warm front propagates along the sample length towards the outlet at the top. Only the right leg is used for the thermal hydraulic measurements. The 21 temperature sensors $\left(T_{11}-T_{17} \ldots T_{31}-T_{37}\right)$ at three rings along the sample (R1, R2 and R3) are shown. The area near the heaters $\mathrm{H} 7, \mathrm{H} 8$ and $\mathrm{H} 9$ is enlarged for clarity. The distance between the heater H0 and the sample inlet is not to scale. The mass flow rate sensor near the sample outlet is not shown. The thermometer $T_{28}$, clamped on sample (not at R2) and used to monitor the cool down, is not shown. . . . . . . . . . . . . 8

4 Sensor ring applied on the LCJ conductor. . . . . . . . . . . . . . . . . 9

5 Details of one half of the sensor ring with 3 Cernox sensors. . . . . . . . . . 9

6 Helium heater $\mathrm{H} 7$ (left) and thermometer $T_{17}$ (right) which are included in the central spiral. . . . . . . . . . . . . . . . . . . . 
$7 \quad$ Example of a typical TH measurement before the temperature calibration, i.e. run $\mathrm{LCJH} 280907, \dot{m}=8 \mathrm{~g} / \mathrm{s}$, heater H8. Both the steady state (first step) and transient heating are shown. The time history of the heating current is shown in the upper-left plot. The time history of the raw temperatures in the rings $\mathrm{R} 1, \mathrm{R} 2$ and R3 are shown in the remaining three plots. The signal $T_{28}$ (lower left plot) is included for completeness but is not used in this analysis. . . . . .

8 Example of a typical TH measurement before the temperature calibration, i.e. run $\mathrm{LCJH} 280907, \dot{m}=8 \mathrm{~g} / \mathrm{s}$, heater H8. Only the steady state (first step) heating is shown. The time history of the heating current is shown in the upper-left plot. The time history of the raw temperatures in the rings R1, R2 and $\mathrm{R} 3$ are shown in the remaining three plots. The signal $T_{28}$ (lower left plot) is included for completeness but is not used in this analysis. . . . . . . . . .

9 Example of a typical calibration run, i.e. LCJH280911, 28 September 2005. The time history of the heating current is shown in the upper-left plot. The time history of the raw temperatures at the rings R1, R2 and R3 are shown in the remaining three plots. The signal $T_{28}$ (lower left plot) is included for completeness but is not used in this analysis. . . . . . . . . . . .

10 Calculation of the calibration parameters. Example in the sensor ring R1 of the run LCJH280911 (28 September 2005). In abscissa of each plot is the raw measurement (e.g. $T_{11}$, top left plot) and in ordinate the reference temperature in the central channel (e.g. $\left.T_{17}\right)$, which is also the resulting calibrated temperature (e.g. $T_{11 \text { cal }}$ ). The linear fit $Y=p_{1}+p_{2} X$ (symbol f) of the experimental data (symbol $\mathrm{m}$ ), as well as the parameters of the linear fit $p_{1}$ (top) and $p_{2}$ (bottom), are shown. . . . . . . . . . . . . . .

11 Result of the temperature calibration in the run LCJH280911 (28 September 2005). The time history of the heating current is shown in the upper-left plot. The time history of the calibrated temperatures at the rings R1, R2 and R3 are shown in the remaining three plots. The signal $T_{17} *, T_{27} *$ and $T_{37} *$ are the raw measurements. . . . . . . . . . . . . . . . . . . . .

12 Result of calibration for the the typical run LCJH280907 ( $\dot{m}=8 \mathrm{~g} / \mathrm{s}$, heater H8), showing only the steady state part of the measurement (first heating step). The time history of the heating current is shown in the upper-left plot. The time history of the calibrated temperatures at the sensor rings R1, R2 and $\mathrm{R} 3$ are shown in the remaining three plots. The signal $T_{17} *, T_{27} *$ and $T_{37} *$ are the raw measurements. The error after re-scaling is $<50 \mathrm{mK} . \ldots \ldots$. . . .

13 Results of run LCJH280907 ( $\dot{m}=8 \mathrm{~g} / \mathrm{s}$, heater H8) after the temperature calibration. The distribution of the calibrated temperatures (sensor ring R1, R2 and R3) is shown along the azimuth, i.e. the jacket circumference, at the end of the first heating step $t_{\text {end }}=153.22 \mathrm{~s}$. In particular, the quantity in ordinate $\left(\mathrm{DT}^{*}\right)$ is the difference between the calibrated temperature and its initial value. The temperatures in the central channel are: $T_{17}=0.0203 \mathrm{~K}$, $T_{27}=0.2979 \mathrm{~K}$ and $T_{37}=0.4607 \mathrm{~K}$ (not a function of the azimuth angle). . . . 
14 Results of run LCJH280907 ( $\dot{m}=8 \mathrm{~g} / \mathrm{s}$, heater H8) after application of the Fast Fourier Transform (FFT). All results are at $t_{\text {end }}=153.22 \mathrm{~s}$. Left plots: the distribution of the calibrated temperatures is shown along the azimuth, i.e. the jacket circumference. In particular, the quantity in ordinate $\left(\mathrm{DT}^{*}\right)$ is the difference between the calibrated temperature and its initial value. The reconstructed function $\left(\mathrm{FFT} 1=\mathrm{frec}_{i}\right.$ ) is shown on the same plot. Right plots: the module of the coefficients of the FFT (see Appendix B). The results are shown at the sensor rings $\mathrm{R} 1, \mathrm{R} 2$ and $\mathrm{R} 3 . \ldots \ldots \ldots$

15 Results of run LCJH270904 ( $\dot{m}=8 \mathrm{~g} / \mathrm{s}$, heater H0) after application of the Fast Fourier Transform (FFT). All results are at $\mathrm{t}_{\text {end }}=185 \mathrm{~s}$. The description of the plots is given in Fig. $14 . \ldots \ldots \ldots \ldots \ldots$

16 Results of run LCJH270905 ( $\dot{m}=8 \mathrm{~g} / \mathrm{s}$, heater H3) after application of the Fast Fourier Transform (FFT). All results are at $t_{\text {end }}=192 \mathrm{~s}$. The description of the plots is given in Fig. $14 . \ldots \ldots \ldots \ldots \ldots$

17 Results of run LCJH280906 ( $\dot{m}=8 \mathrm{~g} / \mathrm{s}$, heater H7) after application of the Fast Fourier Transform (FFT). All results are at $\mathrm{t}_{\text {end }}=127 \mathrm{~s}$. The description of the plots is given in Fig. $14 . \ldots \ldots \ldots \ldots \ldots$

18 Results of run LCJH290904 ( $\dot{m}=8 \mathrm{~g} / \mathrm{s}$, heater H9) after application of the Fast Fourier Transform (FFT). All results are at $\mathrm{t}_{\text {end }}=209 \mathrm{~s}$. The description of the plots is given in Fig. 14. . . . . . . . . . . . . . . .

19 Summary of results of Fourier analysis. Axial temperature distribution $A 1_{i}-$ $\Delta T_{i 7}$ along the coordinate $X *$, at all mass flow rates (e.g. on the top-left plot are the results at $2 \mathrm{~g} / \mathrm{s}$ ). The bottom-left plot shows also the exponential fit (dashed line) for the heater H8 and H7 (details in Fig. 21 and 22). The results of heater $\mathrm{H} 3$ at $4 \mathrm{~g} / \mathrm{s}$ and $6 \mathrm{~g} / \mathrm{s}$ are not consistent with the other results of the measurement set and therefore have been rejected in this report. . . . . . .

20 Summary of results of Fourier analysis. Axial temperature distribution $a b s A 2_{i}$ along the coordinate $X *$, at all mass flow rates (e.g. on the top-left plot are the results at $2 \mathrm{~g} / \mathrm{s}$ ). The bottom-left plot shows also the exponential fit (dashed line) for the heater H8 (details in Fig. 21). . . . . . . . . . . . . . . .

21 Example of exponential fit of the temperature distributions $A 1_{i}-\Delta T_{i 7}$ and $a b s A 2_{i}$ along the coordinate $X *$. Heater $\mathrm{H} 8$ at $8 \mathrm{~g} / \mathrm{s}$ (run LCJH280907). Left plot: measurement, fit and parameters $a$ (left) and $k_{B H}$ (right) are shown. Right plot: measurement, fit and parameters $b$ (left) and $k_{B B}$ (right) are shown. 31

22 Example of exponential fit of the temperature distribution $A 1_{i}-\Delta T_{i 7}$ along the coordinate $X *$. Heater $\mathrm{H} 7$ at $8 \mathrm{~g} / \mathrm{s}$ (run LCJH280906). Measurements, fit and parameters $a$ (left) and $k_{B H}$ (right) are shown. . . . . . . . . . . . 
23 Results of flow splitting in the bundle $(\mathrm{B})$ and hole $(\mathrm{H})$. Velocity and mass flow rate, as well as their ratios, are shown as a function of the total mass flow rate. The nominal friction factors are used, i.e. $f_{B}=0.7 f_{\text {Katheder }}$ and

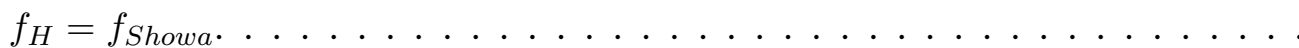

24 Schematic model of the conductor with central channel (hole) and $\mathrm{N}=6$ parallel cooling channels (bundle). The radial heat transfer coefficient between bundle and hole is $h_{B H}$, and the azimuthal heat transfer coefficient between subcables in the bundle is $h_{B B} \ldots \ldots \ldots \ldots \ldots \ldots$

25 Azimuthal heat transfer coefficient between subcables in the bundle, as a function of the total mass flow rate (heater H8). The nominal friction factors are used, i.e. $f_{B}=0.7 f_{\text {Katheder }}$ and $f_{H}=f_{\text {Showa }}$. The fit of the experimental data using the Nield-Bejan expression with the coefficient $C_{T}=0.119$ is also shown (see Eq. 97 in Appendix C). . . . . . . . . . . . . . . . . . . . .

26 Radial heat transfer coefficient between bundle and hole, as a function of the total mass flow rate (heater H7, H8 and H9). The nominal friction factors are used, i.e. $f_{B}=0.7 f_{\text {Katheder }}$ and $f_{H}=f_{\text {Showa }}$. The results of the Long expression are also shown in the range of $h_{\text {extra }}$ between 2 and 5 (see Eq. 16). The results of the PFIS conductor are also included for comparison (PFIS-NW without subcable wraps, PFIS-W with subcable wraps) [4]. . . . . . . . . . . .

27 Results of the sensitivity analysis. The nominal friction factor in the central spiral is used $\left(f_{H}=f_{\text {Showa }}\right)$. The ratio of velocity $v_{H} / v_{B}$, the ratio of mass flow rate $\dot{m}_{H} / \dot{m}_{B}$, the radial heat transfer coefficient $h_{B H}$ and the azimuthal heat transfer coefficient $h_{B B}$ are plotted as a function of the correction of the friction factor in the bundle. The heating is provided by the heater H8. . . . .

28 Results of the sensitivity analysis. The nominal friction factor in the bundle is used $\left(f_{B}=0.7 f_{\text {Katheder }}\right)$. The ratio of velocity $v_{H} / v_{B}$, the ratio of mass flow rate $\dot{m}_{H} / \dot{m}_{B}$, the radial heat transfer coefficient $h_{B H}$ and the azimuthal heat transfer coefficient $h_{B B}$ are plotted as a function of the correction of the friction factor in the hole. The heating is provided by the heater H8. . . . . . .

29 Series of eigenvectors obtained analytically for the case $N=6$, ordered by eigenvalue (from top-left to bottom-right). The two eigenvalues in the center are multiple, and have two associated eigenvectors each. . . . . . . . . . . .

30 Comparison of analytical solution (symbol $x-r e d$ ) and numerical solution (symbol dot-blue). The curve $\mathrm{x}$-green is the average temperature of the bundle. 55 